\title{
LAPTM4B: A Novel Diagnostic Biomarker and Therapeutic Target for Hepatocellular Carcinoma
}

\author{
Rou Li Zhou \\ School of Basic Medical Sciences, Peking University
}

China

\section{Introduction}

The carcinogenesis and progression of hepatocellular carcinoma (HCC) are complicated processes which evolve through distinct stages associated with cumulative genomic alterations, which result in deregulation of proliferation, metastasis and recurrence, leading to poor prognosis. Despite the fact many new therapeutic and diagnostic strategies for cancer have been developed in recent years, the cure rate for HCC has not satisfactorily improved and biomarkers currently used for HCC diagnosis are not satisfactory in either their specificity or sensitivity.

More than one thousand cancer-associated genes and proteins have been identified, but the pathogenesis of hepatocellular carcinoma is still incompletely understood. Identifying the as yet unknown pivotal genes and proteins will be critical for early diagnosis, effective monitoring and novel therapies for this highly aggressive cancer. In previous work we cloned and identified a novel HCC-associated gene (lysosomal protein transmembrane 4 beta, LAPTM4B). Its complementary mRNA is highly expressed in the vast majority of HCC samples as compared with paired noncancerous liver from the same patient (PNL), fetal liver, and normal liver (Shao et al., 2003). Over our past decade studies have demonstrated that the LAPTM4B gene and the LAPTM4B-35 protein which it encodes would be pivotal diagnostic biomarkers and therapeutic targets for hepatocellular carcinoma.

\section{LAPTM4B: A novel gene associated with hepatocellular carcinoma}

\subsection{Identification and cloning of the LAPTM4B gene}

To search for genes involved in deregulation of proliferation and aberrant differentiation for hepatocellular carcinoma, fluorescent-differential display was performed using human liver tissues, including adult liver, fetal liver, HCC and its paired samples of noncancerous liver from the same patient (paired noncancerous liver). A cDNA fragment, which was not a part of any known gene, was found from 110 differential display fragments obtained. This cDNA fragment was highly expressed in HCC, significantly expressed in fetal liver and the paired noncancerous liver, but showed very low expression in normal adult liver (JJ. Liu et al., 2000). Cloning the full length cDNA (NM_018407, ID=55353, Figure 1) harboring this fragment was performed by Expressed Sequence Tag (EST) splicing, 5' rapid amplification 
of cDNA ends (FACE) and reverse transcription-polymerase chain reaction (RT-PCR).

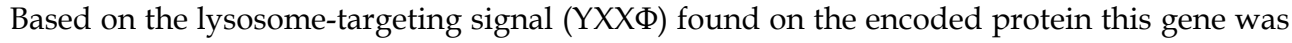
originally designated by the HUGO Gene Nomenclature Committee as "lysosomalassociated protein transmembrane 4 beta" (LAPTM4B) and this designation was then revised as "lysosomal protein transmembrane 4 beta" (LAPTM4B). Importantly, it should be emphasized that reverse-transcription from total RNA to synthesize this cDNA must be conducted at $65^{\circ} \mathrm{C}$ using the ThermoScript RT-PCR Systems (Gibco-BRL) as there is very high content of GC at the $5^{\prime}$ terminus (Shao et al., 2003), otherwise full length LAPTM4B cDNA cannot be obtained. BLAST program analysis shows that the LAPTM4B gene maps to chromosome 8q22.1, spanning at least $50 \mathrm{~kb}$ composed of seven exons separated by six introns, and contains a $951 \mathrm{bp}$ open reading frame (ORF). LAPTM4B mRNA is approximately $2.16 \mathrm{~kb}$ in length and which is consistent with the size of the mRNA observed in Northern blots. Two polyadenylation signal sites, AATAAA and AATTAAA are present at the 3' UTR (Figure 1). The former polyadenylation site (AATAAA) may result in another $1.42 \mathrm{~kb}$ mRNA variant (Shao et al., 2003).

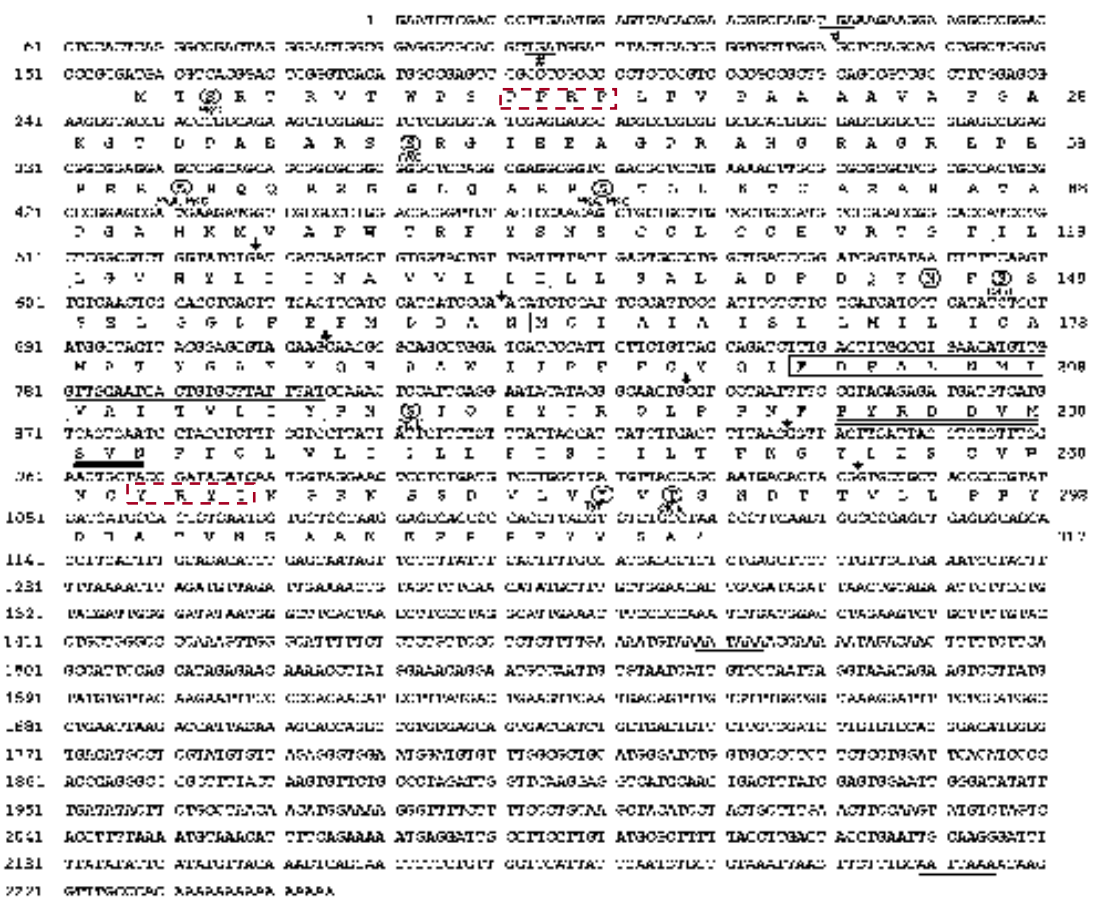

Fig. 1. The nucleotide sequence of LAPTM4B cDNA and the deduced amino-acid sequence. The sequence numbers of the first nucleotide (left) and the final amino acid (right) in each row is indicated, respectively. Exon divisions are indicated by $\mathbf{\nabla}$. The polyadenylation consensus sequences (AATAAA, AATTAAA) are underlined. Transmembrane regions are boxed. The putative $\mathrm{N}$-glycosylation site is marked with a ring of hexagon; potential phosphorylation sites are circled, with the corresponding kinases indicated in italic characters. Stop codon or inframe stop codons are indicated as \# or *. The PPRP and YXXФ motifs are boxed with red line of dashes. (Shao et al., 2003) 


\subsection{Bioinformatics analysis of LAPTM4B proteins}

Bioinformatics shows that the full-length cDNA sequence of LAPTM4B contains two translational initiation codons (ATG) separated by an interval of $273 \mathrm{bp}$ and therefore, encodes two protein isoforms with apparent molecular weights of $35 \mathrm{kDa}$ containing 317 amino acid residues and $24 \mathrm{kDa}$ containing 226 amino acid residues, which are designated LAPTM4B-35 and LAPTM4B-24. LAPTM4B-35 has a pI at 9.07 due to its high content of arginine residues, while LAPTM4B-24 has a $\mathrm{pI}$ at 4.65 resulting from a high content of acidic amino acid residues. Computer analysis shows that LAPTM4B is an integral membrane glycoprotein (Figure 2), with four transmembrane regions, two extracellular domains (EC1 and EC2), one small intracellular loop, together with one $\mathrm{N}$-terminal and one C-terminal tail which reside in the cytoplasm. One potential N-glycosylation site is present in the EC1 domain and several O-glycosylation sites may also be present. LAPTM4B-35 contains six putative intracytoplasmic phosphorylation sites, including one tyrosine phosphorylation site at Try 285, and four N-myristylation sites. Compared to LAPTM4B-24, LAPTM4B-35 has an extra 91 amino acid sequence in its N-terminus that harbors a proline-rich domain, which serves as the binding site of the SH3 domain in some signaling molecules. A conserved YXXф ( $Y$ represents tyrosine, $\phi$ represents a large hydrophobic amino acid, $\mathrm{X}$ represents any amino acid) motif in the C-terminal tail is believed to function as a signal for targeting to lysosomal membranes and sorting.
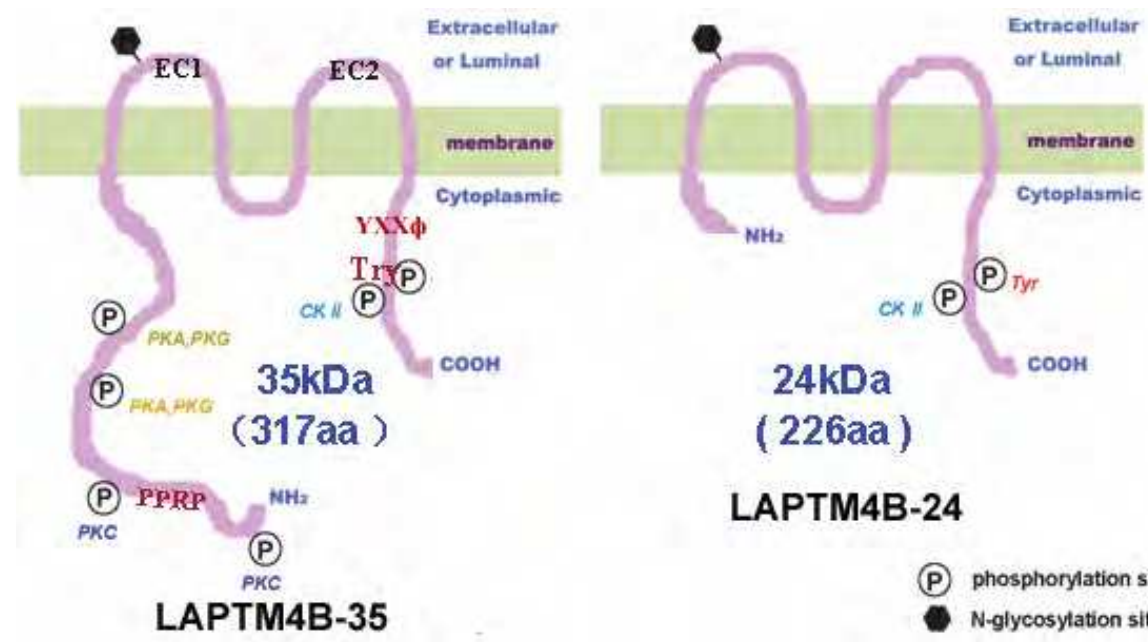

LAPTM4B-24

(P) phosphorylation site

- N-glycosylation site

Fig. 2. Topology of LAPTM4B-35 (left) and LAPTM4B-24 (right) proteins

\subsection{Expression of LAPTM4B mRNA and protein in normal tissues and cancer tissues}

Expression of LAPTM4B mRNA has been evaluated by Northern blot and RT-PCR, and found to be widely expressed in human tissues. Its expression is highest in the heart, skeletal muscle and uterus, and more high in testis and ovary. Expression is moderate in the kidney and pancreas, and low in the liver, spleen and thymus, but is lowest in the lung and peripheral leukocytes (Figure 3a) (Shao et al., 2003; Kasper et al., 2005). High expression is 
also seen in fetal heart, kidney and spleen (Figure 3b). Expression of LAPTM4B mRNA in HCC has been confirmed by Northern blot (Figure 4a), RT-PCR and hybridization in situ (Figure $4 \mathrm{~b}$ ). The frequency of LAPTM4B mRNA over-expression as demonstrated by Northern blot is as high as $87.3 \%$ (Shao et al., 2003); Expression of LAPTM4B-35 and LAPTM4B-24 proteins in tissues have been demonstrated by Western blot and immunohistochemistry (IHC). As shown by Western blot (Figure 5a), LAPTM4B-35 is much more abundant than LAPTM4B-24 in the liver and HCC. In other words, overexpression of LAPTM4B-35 but not LAPTM4B-24 presents in HCC, and the ratio of LAPTM4B-35 to LAPTM4B-24 is thus markedly increased in HCC (X. Liu et al., 2004). The frequency of LAPTM4B-35 up-regulation is also extremely high (Figure 5b), and it has been shown that $\mathrm{T} / \mathrm{N}>1.5$ [T=tumor)/ $\mathrm{N}$ =paired non-cancerous liver] is found in $87.7 \%$ $(57 / 65)$ of HCC, T/N>2 in $76.9 \%(50 / 65)$ of $\mathrm{HCC}$ and T/N $>4$ in $61.5 \%(40 / 65)$ of HCC (Yang et al., 2010a). More importantly, the levels of LAPTM4B-35 in HCC tissues are significantly associated with pathological grades, TNM staging, portal vein invasion and recurrence, but not with age, gender, viral status, tumor size and serum AFP levels (Table 1, Yang et al., 2010a). Moreover, LAPTM4B mRNA and LAPTM4B-35 over-expression also occurs in a wide range of hepatocellular carcinoma- and other cancer-derived cell lines (Shao et al., 2003; XR. Liu et al., 2004).

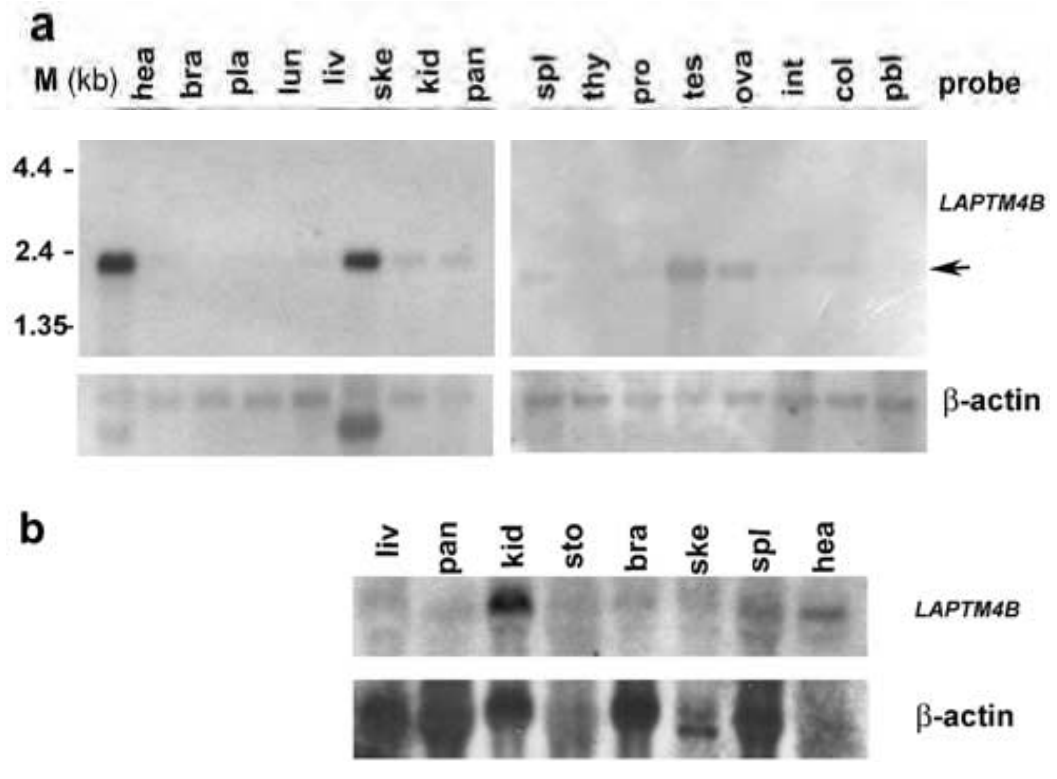

Fig. 3. LAPTM4B mRNA expression in normal human tissues analyzed by Northern blot with a LAPTM4B prob (corresponding to nt 1876-2171 on NM_018407).

(a) Expression of LAPTM4B mRNA in adult human tissues (b) Expression of LAPTM4B mRNA in fetal human tissues (Shao et al., 2003) 

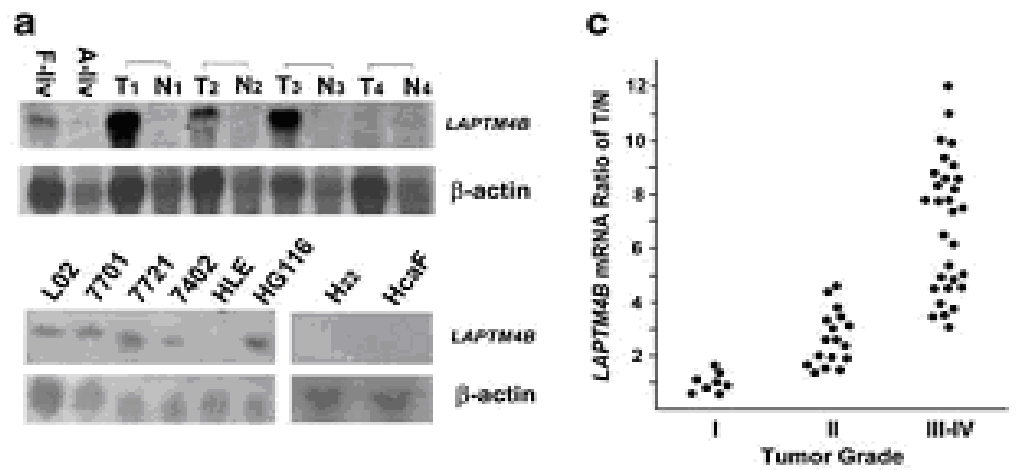

b

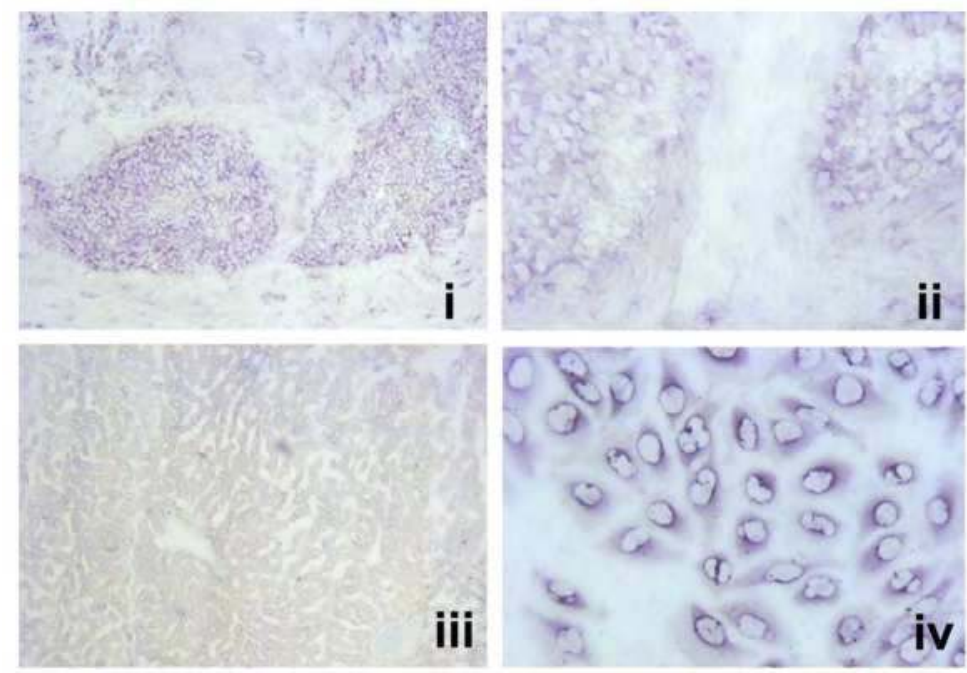

Fig. 4. LAPTM4B mRNA expression in hepatocellular carcinomas (HCCs) tissues and HCCderived cell lines analyzed by Northern blot with a LAPTM4B prob (corresponding to nt 1876-2171 on NM_018407). (a) Northern blot analysis of LAPTM4B. Upper panel: HCC tissues $(\mathrm{T})$, paired noncancerous liver tissues $(\mathrm{N})$, fetal and adult normal liver tissues (F- \& A-liv); Lower panel: The human liver- and HCC-derived cell lines, and mouse HCC-derived cell lines. (b) Hybridization in situ was performed on tissue sections. LAPTM4B mRNA expressions in HCC tissue (i, ii), paired noncancerous tissue (iii), and BEL7402 cells (iv). Positive LAPTM4B mRNA expression was preferentially in hepatic cancerous cells as indicated by blue-purple staining. Original magnification, 40 (i, iii) or 100 (ii, iv). (c) Positive correlation between LAPTM4B mRNA levels and tumor grades. Each spot in the figure represents the ratio of tumor vs. paired noncancerous liver tissue $(T / N)$ of the LAPTM4B mRNA expression evaluated by Northern blot. These spots derived from 55 HCC patients. (Shao et al., 2003) 
A Western Blot

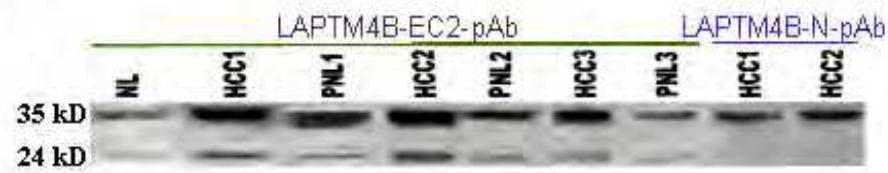

$\begin{array}{lllllllllll}\mathbf{B} & \mathbf{N} & \mathbf{T} & \mathbf{N} & \mathbf{T} & \mathbf{N} & \mathbf{T} & \mathbf{N} & \mathbf{T} & \mathbf{N} & \mathbf{T}\end{array}$
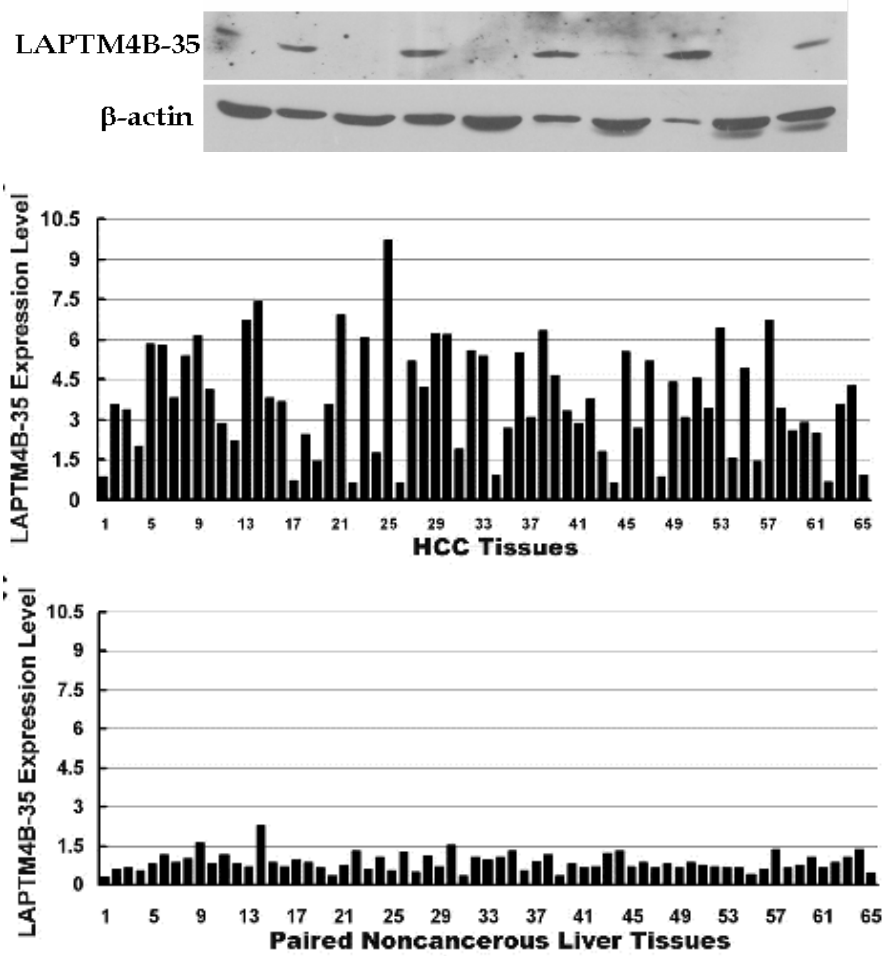

Fig. 5. Expression of LAPTM4B-35 and LAPTM4B-24 protein identified by Western blot in HCCs and paired noncancerous liver tissues (PNL) from the same one patient.

(a) Expression of LAPTM4B-35 and LAPTM4B-24 was evaluated by Western blot with an anti-LAPTM4B-EC2 pAb, which reacts with both LAPTM4B-35 and LAPTM4B-24, and antiLAPTM4B-N-pAb, which reacts with merely LAPTM4B-35. It is shown that LAPTM4B-35 is much more abundant than LAPTM4B-24 in the normal liver (NL) and HCC. (b) Expression of LAPTM4B-35 detected with Anti-LAPTM4B-N-10 pAb. The upper panel shows the expression of LAPTM4B-35 in HCC (T) and paired noncancerous liver tissues $(\mathrm{N})$ tissues. Densitometry was normalized with $\beta$-actin. The middle panel shows the levels of LAPTM4B-35 in HCC tissues derived from 65 patients. The lower panel shows the levels of LAPTM4B-35 in the paired noncancerous liver tissues derived from the same 65 patients. (Yang et al., 2010a). 


\begin{tabular}{|c|c|c|c|c|c|}
\hline \multirow{2}{*}{ Variables } & \multirow{2}{*}{ Patients (n) } & \multicolumn{3}{|c|}{ LAPTM4B-35 expression } & \multirow{2}{*}{$P a$} \\
\hline & & Low & Middle & High & \\
\hline All cases & 65 & 15 & 30 & 20 & \\
\hline Gender & & & & & 0.434 \\
\hline Male & 53 & 11 & 24 & 18 & \\
\hline Female & 12 & 4 & 6 & 2 & \\
\hline Age (years) & & & & & 0.544 \\
\hline$<60$ & 42 & 8 & 21 & 13 & \\
\hline$\geq 60$ & 23 & 7 & 9 & 7 & \\
\hline Cirrhosis & & & & & 0.013 \\
\hline Yes & 44 & 6 & 25 & 13 & \\
\hline No & 21 & 9 & 5 & 7 & \\
\hline Viral status & & & & & 0.766 \\
\hline Hepatitis virus B & 46 & 9 & 23 & 14 & \\
\hline Hepatitis virus $C$ & 12 & 4 & 5 & 3 & \\
\hline Both hepatitis virus B and C & 2 & 0 & 1 & 1 & \\
\hline Non-B, Non-C & 5 & 2 & 1 & 2 & \\
\hline Tumor size & & & & & 0.421 \\
\hline$<5 \mathrm{~cm}$ & 34 & 10 & 15 & 9 & \\
\hline$\geq 5 \mathrm{~cm}$ & 31 & 5 & 15 & 11 & \\
\hline Invasive tumor & & & & & $<0.001$ \\
\hline Yes & 17 & 0 & 4 & 13 & \\
\hline No & 48 & 15 & 26 & 7 & \\
\hline $\begin{array}{l}\text { Histopathological } \\
\text { differentiation }\end{array}$ & & & & & $<0.01^{b}$ \\
\hline Well Differentiation (WD) & 18 & 6 & 8 & 4 & \\
\hline Middle Differentiation (MD) & 24 & 7 & 11 & 6 & \\
\hline Poor Differentiation (PD) & 23 & $2^{*}$ & 11 & $10^{*}$ & \\
\hline Serum AFP level & & & & & 0.517 \\
\hline$<25 \mathrm{ng} / \mathrm{ml}$ & 32 & 6 & 17 & 9 & \\
\hline$\geq 25 \mathrm{ng} / \mathrm{ml}$ & 33 & 9 & 13 & 11 & \\
\hline TNM stage & & & & & $<0.001$ \\
\hline I & 16 & 11 & 3 & 2 & \\
\hline II & 21 & 2 & 14 & 5 & \\
\hline III-IV & 28 & 2 & 13 & 13 & \\
\hline $\begin{array}{l}a \text { : Chi-square test; } \\
b: \mathrm{p}<0.01 \text { : Low expression vs. High }\end{array}$ & ession for PD & & & & \\
\hline
\end{tabular}


In addition, over-expressions of LAPTM4B mRNA and LAPTM4B-35 protein have also been found in other solid cancers. Kasper et al. (2005) reported that LAPTM4B mRNA tested by Northern Blot is over-expressed in $88 \%$ of lung cancer, $50.9 \%$ of breast cancers, $67 \%$ of colon cancers, $68 \%$ of uterus cancers, and $37 \%$ of gastric cancers. Peng et al. (2005) reported that LAPTM4B-35 protein as evaluated by immuno-histochemistry (IHC) is highly expressed in lung cancer, stomach cancer, colon cancer and breast cancer. Subsequently, the frequency of over-expression of LAPTM4B-35 in more cases of various cancers was determined, and was found in $76 \%$ of gallbladder cancers (Zhou et al., 2007), $72 \%$ of cholangiocarcinomas (Zhou et al.,. 2008), 63.5\% of ovarian cancers (Yang et al., 2008; Yin et al., 2010), 72.57\% of cervical cancers (Meng et al., 2010a) and 70.91\% of endometrial cancers (Meng, et al., 2010b). However, the mechanism for over-expression of LAPTM4B in HCC and other cancers has not as yet been completely elucidated. Mutation and demethylation of LAPTM $4 B$ gene has not been found. Nevertheless, it has been reported in a large number of articles that the chromosome 8q region harboring LAPTM4B gene is amplified as shown by fluorescence in situ hybridization (FISH) or gained as shown by comparative genomic hybridization (CGH) in both hepatoblastoma and hepatocellular carcinoma (Buendia et al., 2002; Longerichet al., 2011; Marchio et al., 1997). More precisely, it has been recently reported that chromosome 8q 22 where the LAPTM4B gene localizes is amplified or gained in breast cancer (Hu et al., 2009; Y. Li. et al., 2010). Therefore, gene amplification may be the cytogenetic basis of LAPTM4B over-expression. However, the genomic DNA copy number alterations of most genes in general do not appear to parallel corresponding transcriptional expression (Huang et al., 2006). It is reasonable to propose that transcriptional up-regulation by transcription factors and/or microRNAs may also contribute to LAPTM4B-35 over-expression in cancers. A more in-depth analysis will be required to clarify these points.

\subsection{LAPTM4B alleles and their significance in susceptibility for hepatocarcinogenesis}

Two alleles of the LAPTM $4 B$ gene have been identified in our laboratory and designated LAPTM $4 B{ }^{*} 1$ and ${ }^{*} 2$. Allele ${ }^{*} 2$ differs from allele ${ }^{*} 1$ in that it contains an extra tandemly arranged 19-bp (gcttggagctccagcagct) sequence at the 5'UTR in the first exon. A PCR-based method was established for genotyping of this gene using the primers $5^{\prime}$ GCCGACTAGGGGACTGGCGGA 3' and 5' CGAGAGCTCCGAGCTTCTGCC $3^{\prime}$ to amplify the partial sequence of exon 1 , and using genomic DNA as the template. To investigate the relationship between the allelic variants of $L A P T M 4 B$ and the susceptibility to HCC or esophageal squamous cell carcinoma (ESC), patients with HCC or ESC, and two control groups of normal individuals from the corresponding regions were analyzed. Significant differences in the frequency of genotype $L A P T M 4 B^{*} 2 / 2$ were found in patients with HCC $(17.4 \%)$ as compared with the controls $(10.2 \%)(\mathrm{p}<0.05)$, indicating that individuals with the ${ }^{*} 2 /{ }^{*} 2$ genotype are more susceptible to HCC than ${ }^{*} 1 /{ }^{*} 1$ and ${ }^{*} 1 /{ }^{*} 2$ individuals (Table 2). However, no difference was observed in the frequencies of LAPTM4B genotypes in patients with ESC as compared with corresponding controls. These results suggest that allele ${ }^{*} 2$ may be associated specifically with susceptibility to HCC. In conclusion, our data suggest that $L A P T M 4 B^{*} 2 /{ }^{*} 2$ is associated with susceptibility to HCC and the LAPTM $4 B$ genotype provides a new means for screening for people who are susceptible to primary hepatocellular carcinoma. This may be of importance for the assessment and prevention of developing hepatocellular carcinoma in high risk populations, in particular for the patients with liver cirrhosis of small liver nodule from HBV and HCV chronic infection (Shao et al., 
unpublished data). In addition, allele $* 2$ has been found to be associated with increased risk of lung cancer (Deng et al., 2005), stomach cancer (Liu et al., 2007), cervical carcinoma (Meng et al., 2011) and colon cancer but not of rectal cancer (Cheng et al., 2008). These results indicate that $L A P T M 4 B{ }^{*} 2$ is also a potential risk factor for the development of some solid cancers.

\begin{tabular}{lccc}
\hline & \multicolumn{2}{c}{$\mathrm{N}(\%)$} & \\
\cline { 2 - 3 } & $\begin{array}{c}\text { Controls } \\
(\mathrm{n}=206)\end{array}$ & $\begin{array}{c}\text { HCC } \\
(\mathrm{n}=184)\end{array}$ & P value \\
\hline LAPTM4B genotype & $97(47.1)$ & $77(41.8)$ & \\
$* 1 / 1$ & $88(42.7)$ & $75(40.8)$ & \multirow{2}{*}{$0.038^{\mathrm{a}}$} \\
$* 1 / 2$ & $21(10.2)$ & $32(17.4)$ & \\
$* 2 / 2$ & & & \\
Allele frequency & 0.68 & 0.62 & \\
$* 1$ & 0.32 & 0.38 & \\
$* 2$ & & \\
\hline
\end{tabular}

a : when compared with the combined frequency of $1 / 1$ and $1 / 2$;

OR: $1.855,95 \%$ CI: $1.027-3.348$

Table 2. Distribution of LAPTM4B genotypes in HCC and Controls

\subsection{LAPTM family}

In addition to LAPTM4B, LAPTM4A (Hogue et al., 1996) and LAPTM5 (Adra et al., 1996) are also members of lysosome-associated protein transmembrane (LAPTM) family. LAPTM4A (27kDa) shows a $46 \%$ homology with LAPTM4B in amino acid sequences. In comparison with LAPTM4B-35 containing 317 amino acid residues, LAPTM4A containing 233 amino acid residues and LAPTM5 containing 262 amino acid residues both are of the Nterminus-truncated molecules of LAPTM family. The three members in the LAPTM family all localize at late endosomes and lysosomes, and play an important role in lysosomal function, including transporting structurally unrelated amphiphilic molecules between cytosol and lysosomes, and are involved in autophagocytosis. Moreover, LAPTM4B, LAPTM4A and LAPTM5 interact and co-localize with mucolipin 1, which is a lysosomal ion channel that belongs to the transient receptor potential (TRP) superfamily and their loss-offunction mutations result in mucolipidosis type IV (MLIV), a lysosomal storage disorder characterized by severe mental and psychomotor retardation (Vergarajauregui et al., 2011). LAPTM4A is involved in the subcellular compartmentalization of diverse hydrophobic small molecules and contributes to the inherent drug sensitivity or resistance of the mammalian cell (Hogue et al., 1999). The LAPTM5 gene maps to chromosome 1p34 and is expressed mainly in hematopoietic cells and immune cells (Adra et al., 1996). LAPTM5 protein physically interacts with the $\mathrm{B}$ cell receptor (BCR) complex and promotes its degradation in the lysosomal compartment in mouse $B$ cells, and thus negatively regulates cell surface BCR levels and B cell activation (Ouchida, 2010). The expression of LAPTM5 gene is usually down-regulated through DNA methylation in a neuroblastoma cell-specific manner, while over-expression of this gene may induce spontaneous regression of neuroblastomas. It is believed that caspase-independent lysosomal cell death due to lysosomal destabilization resulted from LAPTM5 up-regulation is closely associated with the spontaneous regression (Inoue et al., 2009). 
Notably, LAPTM family shows similarity in some functional characteristics to "tetraspanin" family, but difference in the structural characteristics, including lacking some conserved amino acid residues and having a smaller EC2 domain. (Maecker et al., 1997; Martin, 2005).

Evolutionarily, LAPTM is an ancient and conserved gene family in mammalian species as well as in lower eukaryotic organisms, including zebrafish (Danio rerio) and Drosophila. The phylogenic tree shown in Figure 6 is constructed according to multiple sequence alignment results. It shows that LAPTM4B and the other two genes in the LAPTM family, LAPTM4A and LAPTM5, are distributed in clusters. Human LAPTM4B shows the closest similarity to Bos Taurus, and also shows some similarity to its zebrafish homolog. In addition, LAPTM4B is more related to LAPTM4A than LAPTM5.

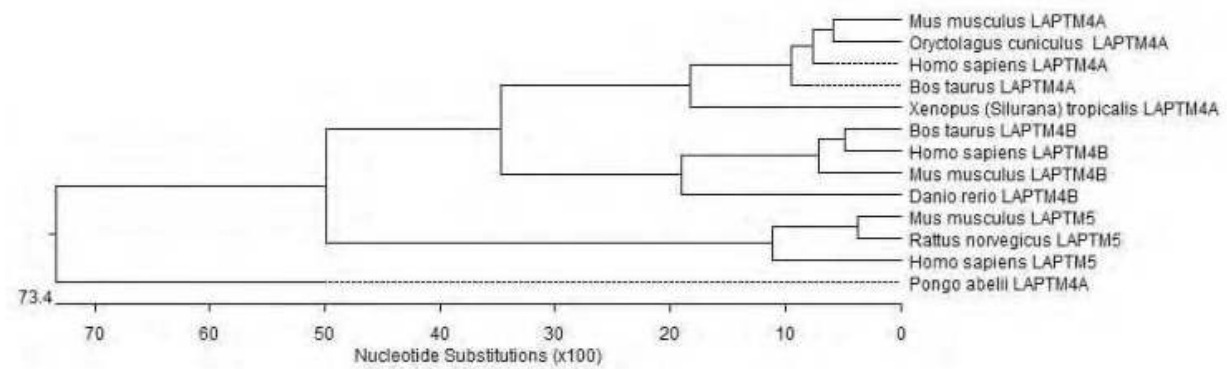

Fig. 6. The evolutionary lineage tree for LAPTM family (This figure was prepared by Shuang Shi)

\section{Functions of LAPTM4B-encoding proteins and the mechanisms thereof}

Functional studies of LAPTM4B-encoding proteins and the mechanisms thereof have been performed via transgenic and gene knockdown techniques. LAPTM4B cDNA is introduced into plasmids with or without a FLAG tag, or into an E1/E3-deleted replication-deficient adenovirus type 5 (Ad5) vector. The LAPTM4B-35 expressing plasmids and cells are all designated in our publications as "AF" if they have a FLAG tag or as "AE" if they do not have a FLAG tag. Similarly, LAPTM4B-24 expressing plasmids and cells are designated as "BF" or "BE". LAPTM4B-35 knockdown is performed by transfection of shRNA-expressing plasmids (Yang et al., 2010c). These functional studies of LAPTM4B-35 and LAPTM4B-24 have determined that these two isoforms of LAPTM4B proteins have antagonistic functions, namely up-regulation of LAPTM4B-35 and LAPTM4B-24 respectively promotes and suppresses hepatocarcinogenesis and metastasis.

\subsection{Over-expression of LAPTM4B-35 promotes carcinogenesis and metastasis of hepatocellular carcinoma}

The effect of LAPTM4B over-expression on carcinogenesis was first explored using mouse NIH3T3 fibroblast cell line (He et al., 2003). Two cell lines stably over-expressing both LAPTM4B mRNA and LAPTM4B-35 were obtained from transfection of LAPTM4B cDNA integrated in plasmids. The LAPTM4B-35 over-expressing NIH3T3-AE cells generated a palpable mass by day 7 in all sites of inoculation in NIH3T3 mice, while in the control groups inoculated with NIH3T3-Mock cells or NIH3T3 parent cells, no tumor appeared 
within an 80 day period following inoculation. Half of the tumor masses generated from LAPTM4B-overexpressing NIH3T3-AE cells showed growth and were histochemically identified as malignant fibrosarcoma; the other half of these masses regressed and were finally identified as liquid lymphoid tissue. Further, the L02 cell line which originated from normal human liver was then used to generate a LAPTM4B-35 over-expressing cell model by infection with replication deficient adenovirus Ad-AE containing LAPTM4B-35 full length cDNA (Lily et al., 2011). Inoculation of LAPTM4B-35 over-expressing L02-AE cells can result in rapidly growing carcinoma xenografts in 100\% (6/6) of inoculated sites in the left axilla of nude mice (Figure $7 \mathrm{a}$ and $7 \mathrm{~b}$ ) and shorten their live span (Figure $7 \mathrm{~d}$ ), as

A
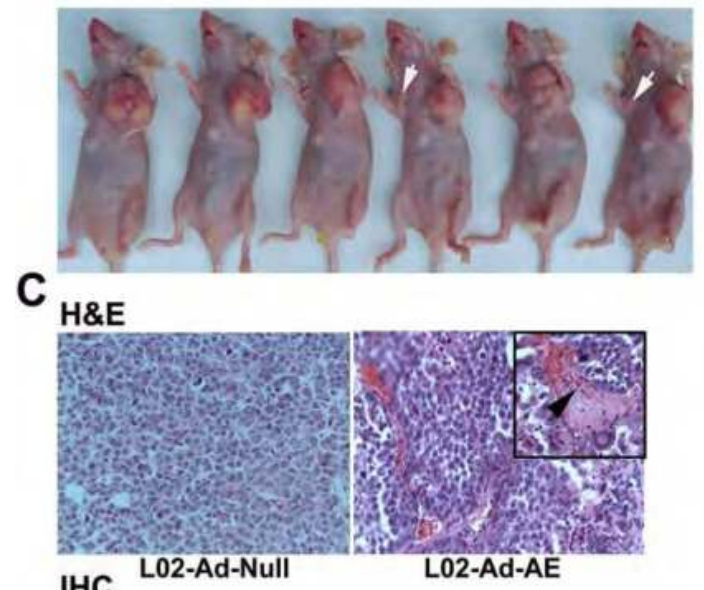

IHC

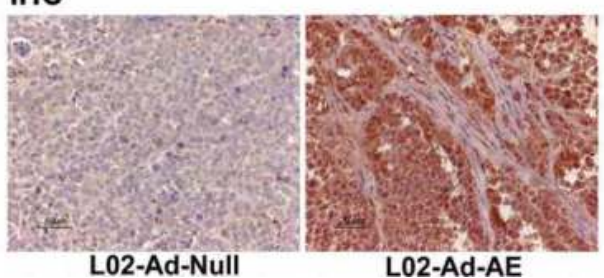

B

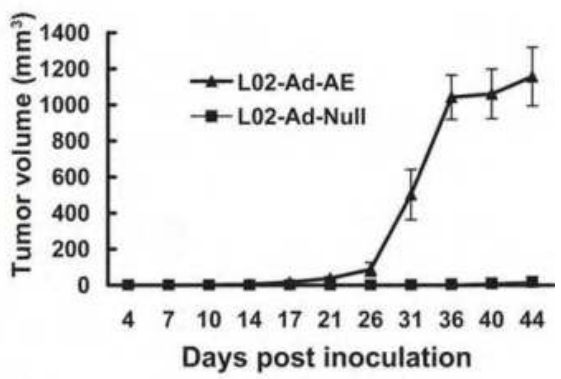

D

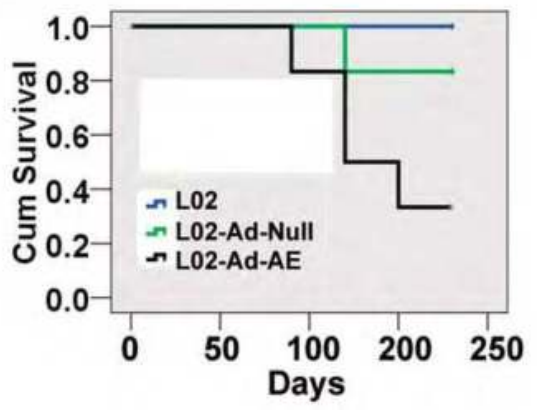

Fig. 7. Upregulation of LAPTM4B-35 promotes tumorigenesis in nude mice. (a) Xenograft tumors formed from L02-Ad-AE cells, in which LAPTM4B-35 is overexpressing, and tumors formed from L02-Ad-Null cells which are indicated by white arrows. (b) Tumor growth curves from L02-Ad-AE cells and L02-Ad-Null cells. (c) Top panel: H\&E staining of tumor formed from L02-Ad-Null cells (left) and L02-Ad-AE cells (right). Black arrows indicate the cancer cells in blood vessel. Bottom panel: immunohistochemical evaluation of LAPTM4B35 expression in xenografts. Tumors derived from L02-Ad-AE cells showed high expression of LAPTM4B-35 (right), but tumors derived from L02-Ad-Null cells showed very low expression of LAPTM4B-35 (left). An anti-LAPTM4B-N10 pAb, which specifically reacts with LAPTM4B-35, was used for IHC (original magnification 200X). (d) Kaplan-Meier survival curves plotted with SPSS $16.0(n=6)$. LAPTM4B-35 up-regulation shortened the live span of mice challenged with L02-Ad-AE cells, as compared to the control group infected by Ad-null, the empty Ad vectors. P=0.049 L02-Ad-AE versus L02-Ad-Null. (Lily et al., 2011). 
A

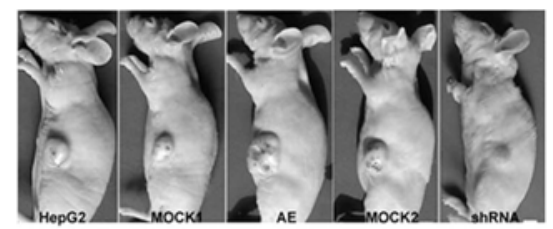

D
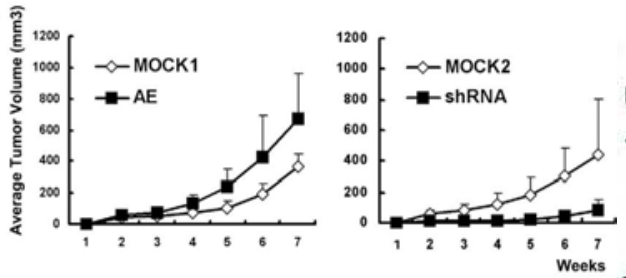

C
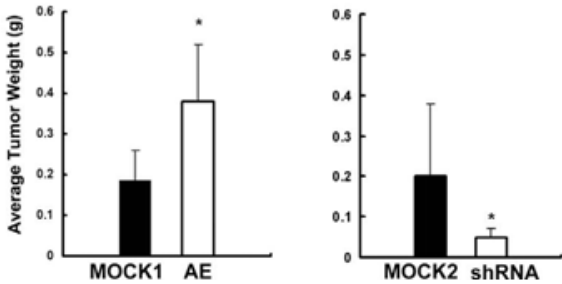

BEL7402

-MOCK2

Lymph node Lung

BEL

BEL7402

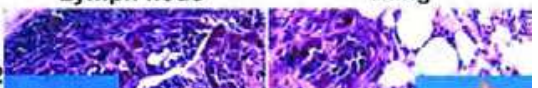

MOCK1
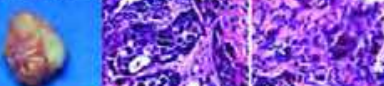

Hesing

$\rightarrow$
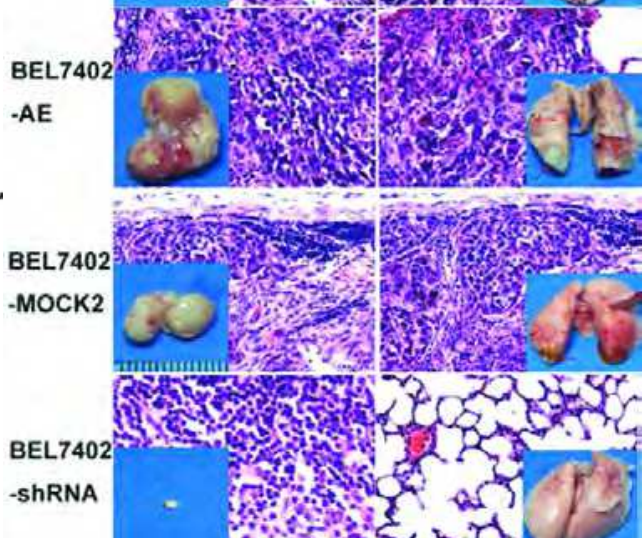

Fig. 8. LAPTM4B-35 promotes tumor growth and metastasis in nude mice. (a)

Representative xenograft tumors formed from HepG2-AE cells over-expressing LAPTM4B35 and HepG2-shRNA cells with LAPTM4B-35 knockdown, and corresponding controls (HepG2-mock1 cells and HepG2-mock2 cells), (b) Tumor growth curves from HepG2-AE and HepG2-mock1 control cells (left), and from HepG2-shRNA and HepG2-mock2 control cells (right). (c) Average tumor weight in nude mice sacrificed 7 weeks after inoculation with HepG2-AE and HepG2-mock1 control cells (left), and with HepG2-shRNA and HepG2mock2 control cells (right). $\mathrm{n}=6$. (P < 0.001: HepG2-AE vs. HepG2-mock1 and HepG2shRNA vs. HepG2-mock2). For all data the mean and standard deviation represent the average of three independent experiments. (d) LAPTM4B-35 promotes tumor metastasis in nude mice: Macroscopic photos and microscopic photos shown by H\&E staining of tumor metastases in lymph nodes and lungs of mice subcutaneously inoculated with BEL7402-AE cells (upper panels), BEL7402-shRNA cells (lower panels) or corresponding control cells. (Yang et al., 2010c)

compared to the control group infected by Ad-null, in which very small tumors were formed in the right axilla of nude mice (Figure $7 \mathrm{a}$ as indicated by the white arrows). The xenografts, which were generated from L02-AE cells, expressed high levels of LAPTM4B-35 as shown by immunohistochemistry (Figure 7c bottom right panel), and these tumors were very well vascularized and tumor blood vessels showed invasion of cancer cells by H\&E staining (Figure 7c top right panel). These findings demonstrated that LAPTM4b-35 overexpressing liver cells have potential for hepatocarcinogenesis and metastasis. In addition, the HepG2 cell line which originated from human hepatoblastoma and the BEL7402 cell line which originated from human hepatocellular carcinoma were used for generating upregulated and down-regulated cell models to study the effects of LAPTM4B-35 on tumor 
growth and metastasis (Yang et al., 2010c). The xenografts in nude mice originating from HepG2-AE and BEL7402-AE cells, in which LAPTM4B-35 has been up-regulated, grow significantly faster (Figure 8a, 8b and 8c left pannel) and with greater numbers of more widespread metastases in the lymph nodes and lungs than the control group transfected by Mock (empty) plasmids (Figure 8d upper panels). Conversely, the xenografts in nude mice originating from HepG2-RNAi and BEL7402-RNAi cells, in which endogenous LAPTM4B35 has been knocked down, grow significantly slower (Figure 8a, 8b and 8c right panel). At the same time, metastases in the lymph nodes and lungs in the HepG2-shRNA and BEL7402-shRNA groups were also fewer and smaller than in the corresponding control groups (Figure 8d lower panels). Overall these experiments demonstrate that up-regulation of LAPTM4B-35 promotes HCC growth and metastasis, while down-regulation has an inhibitory effect.

\subsection{Over-expression of LAPTM4B-35 induces deregulation of proliferation}

Promotion and inhibition of proliferation by LAPTM4B-35 up-regulation and downregulation respectively has been demonstrated conclusively using L02-AE, BEL7402-AE, HepG2-AE and HLE-AE cell lines, together with BEL7402-RNAi and GepG2-RNAi cells (L. Li et al,. 2011; XR. Liu et al., 2009; H.Yang et al., 2010c). Except the L02 cell line was originally derived from normal liver, the BEL7402, HepG2 and HLE cell lines were all originally derived from human HCCs. It is noteworthy that up-regulation of LAPTM4B-35 not only accelerates cell proliferation (Figure 9a, 9b and 9c), but also induces deregulation of proliferation, which is a characteristically neoplastic phenotype. HLE-AE, BEL7402-AE and HepG2-AE cells in which LAPTM4B-35 has been up-regulated, generate colonies which are significantly larger and greater in number; whereas HepG2-RNAi and BEL7402-RNAi cells in which the endogenous LAPTM4B-35 has been down-regulated produce markedly smaller and fewer colonies in soft agar (Figure 9d), respectively demonstrating enhancement and diminution of anchorage-independent growth which is a fundamental criterion for evaluation of proliferative deregulation in malignant transformation (XR.Liu et al., 2009; H.Yang et al., 2010). In addition, growth of HLE-AE cells is less dependent on exogenous growth factors derived from serum supplement as compared with control HLE-MOCK cells, representing auto-secretion of growth factor by LAPTM4B-35 overexpressing cells which is another criterion of proliferative deregulation and malignant transformation (XL.Liu et al., 2009).

Moreover, LAPTM4B-35 over-expression alters not only the proliferation-associated malignant cellular phenotype, but also the proliferation-regulatory proteins encoded by oncogenes and tumor suppressor genes (He et al., 2003; XR. Liu et al., 2009; H. Yang et al., 2010c). We have found that cell cycle-promoting proteins including cyclin D1, cyclin E and $\mathrm{p}-\mathrm{Rb}$ are up-regulated in LAPTM4B-35 over-expressing HepG2-AE, BEL7402-AE and HLEAE cells. At the same time, the transcription factors c-Myc, c-Fos and c-Jun that positively regulate expression of cyclins, are also significantly up-regulated. Conversely, the cell cycleinhibiting proteins of cyclin-dependent kinase inhibitor (CKI) family, including p16, p21 and in particular p27 are markedly down-regulated in these LAPTM4B-35 over-expressing HCC cell lines. At the same time, knockdown of endogenous LAPTM4B-35 gives reverse effects (Figure 10). These studies serve to identify the molecular basis for deregulated malignant proliferation resulting from LAPTM4B-35 over-expression. 

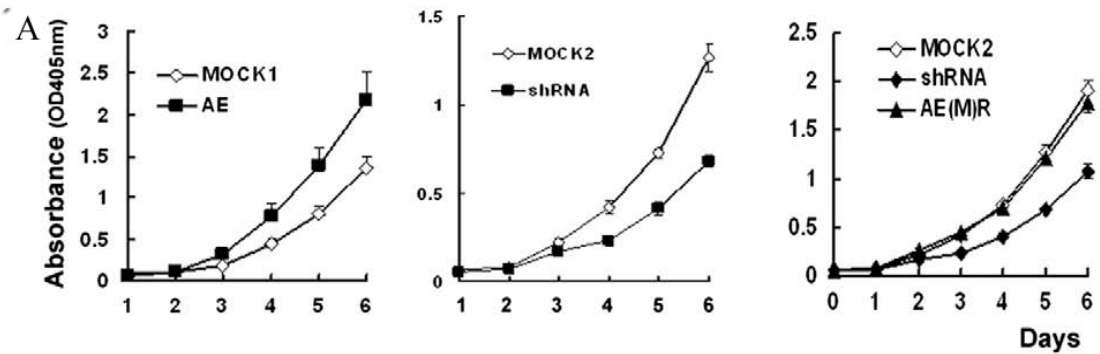

B

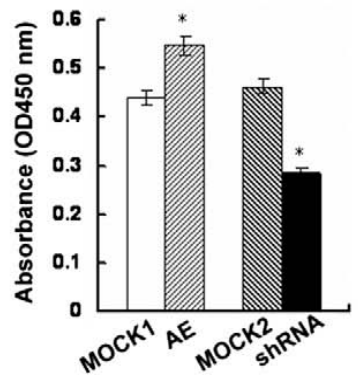

$\mathrm{D}$

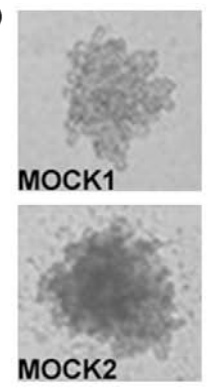

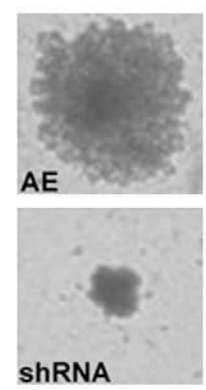

$\mathrm{C}$

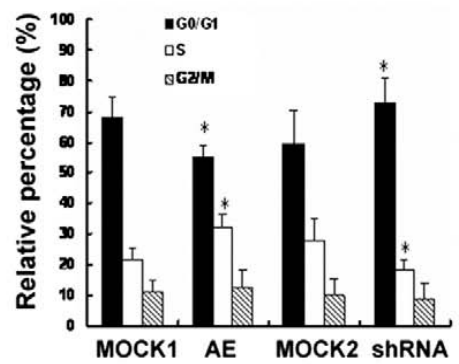

Fig. 9. Over-expression of LAPTM4B-35 promotes cell proliferation and induces deregulation of proliferation. (a) Growth curves determined by MTT assay. Left panel: overexpression of LAPTM4B-35 promotes rapid increase in cell viability/proliferation compared with the control. Middle panel: knockdown of LAPTM4B-35 inhibits increase in cell viability/proliferation as compared with the control. Right panel: restoration of LAPTM4B-35 expression in HepG2(M)R cells, in which the RNAi target was mutated, so that the siRNA produced from shRNA can not bind to the target mRNA, reverses inhibition of cell viability/proliferation resulting from knockdown of LAPTM4B-35. (b) DNA synthesis analyzed by BrdU incorporation assay. P < 0.05: HepG2-AE vs. HepG2-mock1, and HepG2shRNA vs. HepG2-mock2. (c) Cell cycle analyzed by FACS. P < 0.05: HepG2-AE vs. HepG2mock1, and HepG2-shRNA vs. HepG2-Mock2. (d) LAPTM4B-35 promotes colony formation in soft agar. Left panel: overexpression of LAPTM4B-35 promoted colony formation (upper); knockdown of endogenous LAPTM4B-35 inhibited colony formation (down). Right panel: a histogram showing colony numbers larger than $50 \mu \mathrm{m}$ that were counted 4 weeks after seeding. *P < 0.05: HepG2-AE vs. HepG2-mock1; **P < 0.001: HepG2-shRNA vs. HepG2mock2. (H. Yang et al., 2010c) 


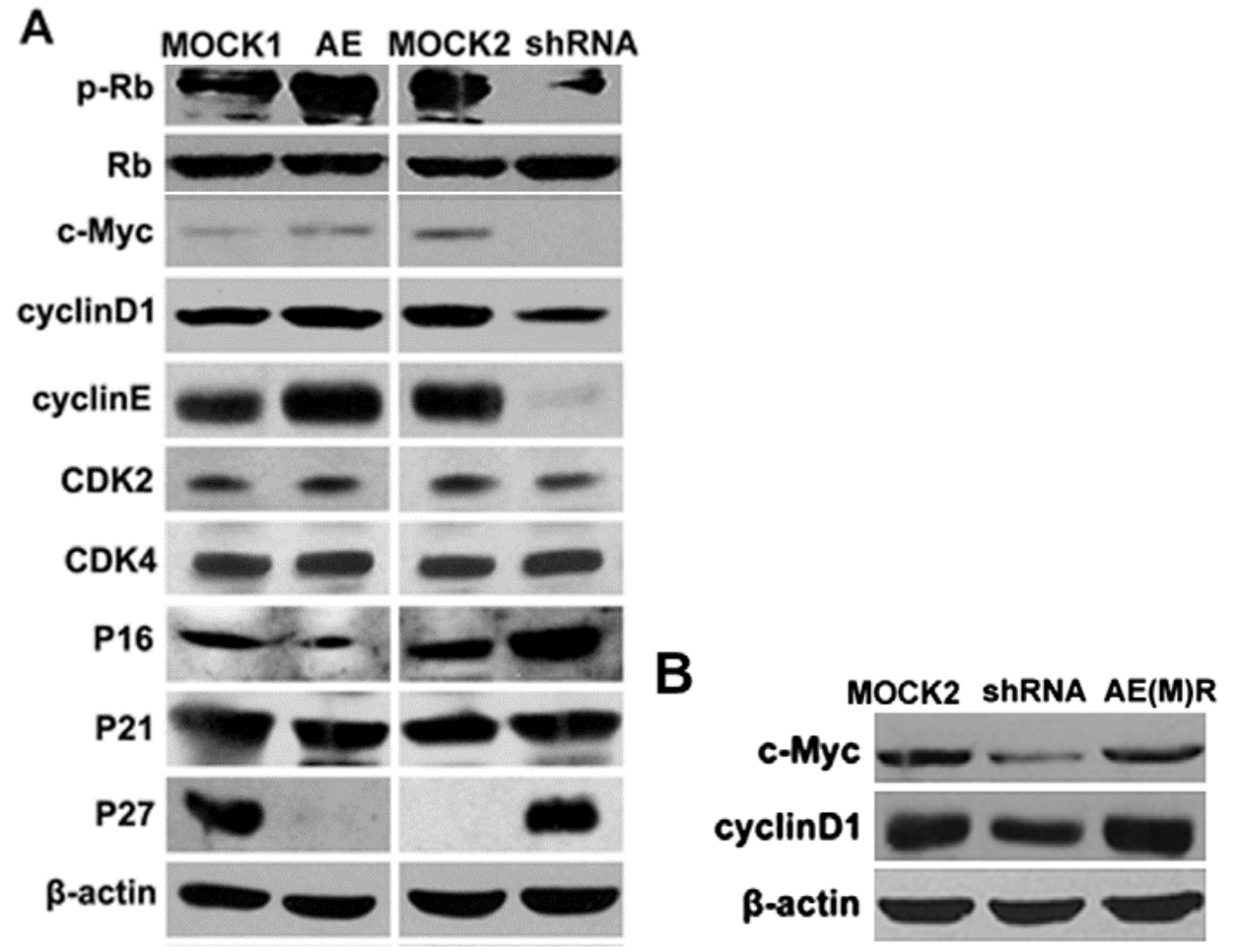

Fig. 10. LAPTM4B-35 alters levels of proliferation-regulating proteins analyzed by Western blot: (a) Level of p-Rb, c-Myc, cyclinD1 and cyclinE protein was significantly increased by over-expression and decreased by knockdown of LAPTM4B-35, respectively. Conversely, level of tumor suppressor gene products, p16 and p27, was significantly decreased by over-expression and increased by knockdown of LAPTM4B-35, respectively. But level of CDK2, CDK4 and p21 was not altered, (b) Restoration of LAPTM4B-35 expression in HepG2-AE(M)R cells reversed the decrease in c-Myc and cyclinD1 proteins resulting from knockdown of endogenous LAPTM4B-35 in HepG2-shRNA cells. (H. Yang et al., 2010c).

\subsection{Over-expression of LAPTM4B-35 enhances resistance to induced apoptosis}

Resistance to apoptosis is one of fundamental characteristics of cancer cells. LAPTM4B-35 over-expressing L02-AE, HepG2-AE and BEL7402-AE cells show marked resistance to druginduced apoptosis (Figure 11-13). However, down-regulation of endogenous LAPTM4B-35 can restore sensitivity of cancer cells to drug-induced apoptosis (L.Li et al., 2010; H.Yang et al., 2010c). In addition, up-regulation of LAPTM4B-35 inhibits activation of the apoptosis executive caspase 3, up-regulates the anti-apoptotic gene bcl-2, bcl-xL and phosphorylated Bad, and also down-regulates the pro-apoptotic gene Bax and Bid (Figure 12, L.Li et al., 2011; L. Zhou, 2011). Moreover, PI3K/AKT, a fundamental signaling pathway for cell survival is activated by up-regulation of LAPTM4B-35, and is inhibited by down-regulation 
of endogenous LAPTM4B-35 (L.Li et al., 2010, 2011; H. Yang et al., 2010c). This data serves to establish the cellular and molecular basis of resistance to apoptosis promoted by overexpression of LAPTM4B-35.

A

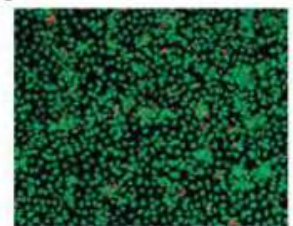

L02-Ad-Null

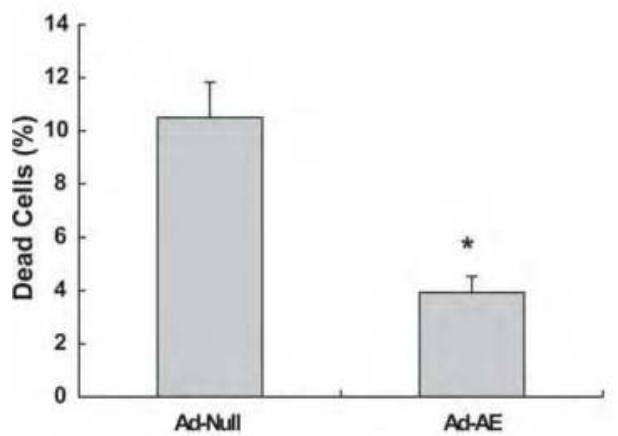

C

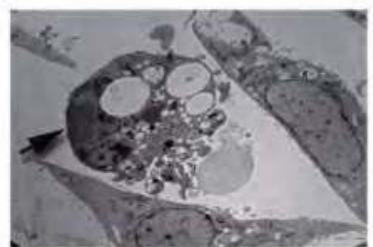

L02-Ad-Null

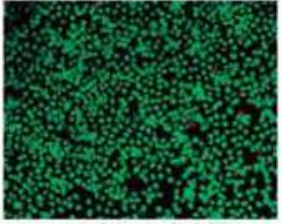

L02-Ad-AE
B
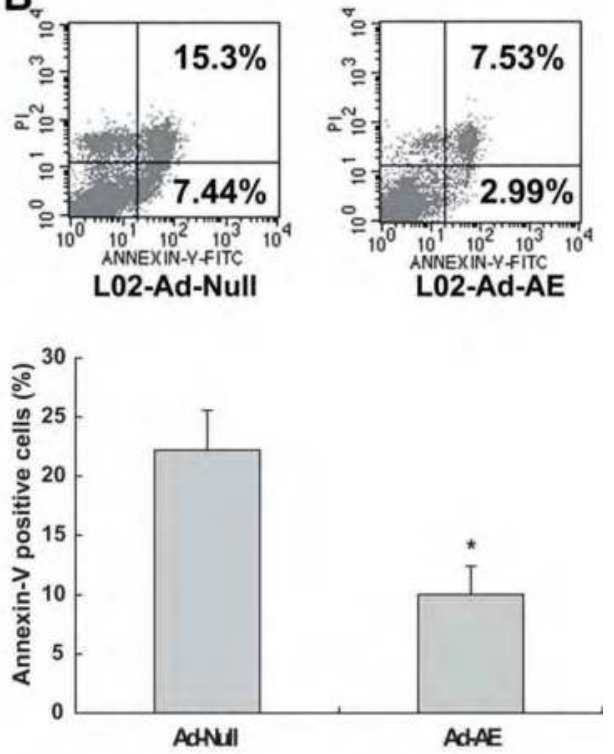

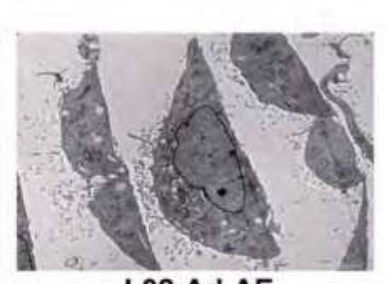

L02-Ad-AE

${ }^{*} \mathrm{P}<0.05$ : Ad-Null vs. Ad-AE

Fig. 11. Up-regulation of LAPTM4B-35 protects L02-AE cells from adriamycin induced apoptosis. (a) LIVE/DEAD Viability/Cytotoxicity Kit assay. Green-stained cells are viable cells; cells with red stained nuclear are late apoptotic and dead cells. The LAPTM4B-35 overexpressing L02-Ad-AE cells show less apoptotic cells as compared with L02-Ad-Null control cells. (b) Upper panel: Flow cytometry analysis of apoptosis by Annexin V and PI staining. The number of apoptotic L02-Ad-AE cells appeared in early-, late-phase of apoptosis and dead phase were all less than L02-Ad-Null cells. Lower panel: The histograms showing the cell percentage of dead cells (left) and Annexin V-positive cells in early plus late apoptotic phases (right). (c) Apoptosis shown by transmission electron microscopy. Black arrow indicates the apoptotic L02-Ad-Null control cell induced by adriamycin; this was not seen in L02-Ad-AE cells (L. Li et al., 2011). 


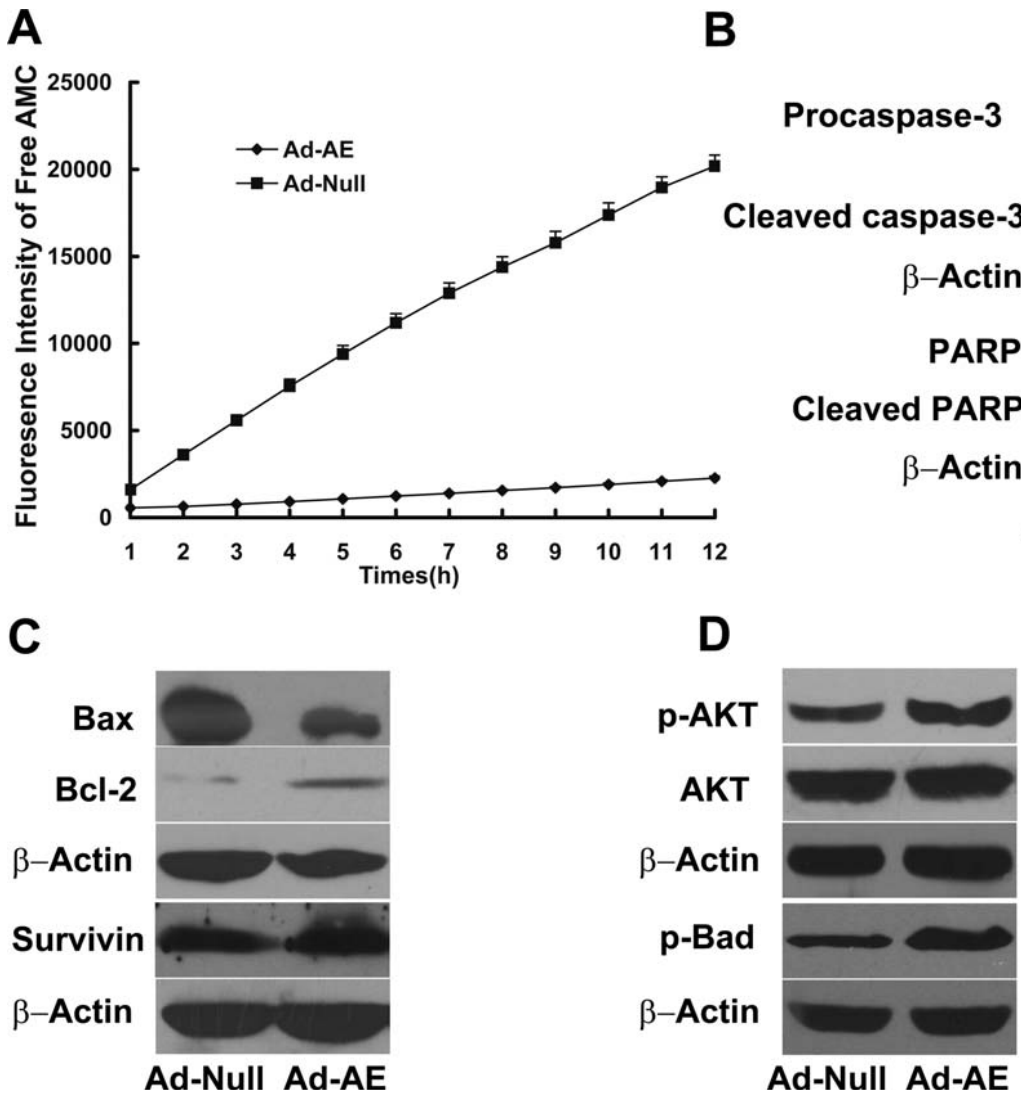

Fig. 12. Up-regulation of LAPTM4B-35 effects activation or expression of apoptosisassociated molecules in L02-AE cells. (a) Inhibition of caspase-3 activation in LAPTM4B35 over-expressing L02-Ad-AE cells measured by the DEVD cleavage assay. Datum in each point represents the mean SD of three independent experiments. ${ }^{* * *} \mathrm{P}<0.001$ : L02Ad-AE vs. L02-Ad-Null. (b) Cleavage of procaspase-3 and PARP was inhibited by upregulation of LAPTM4B-35, demonstrating the inhibition of apoptosis. (c) Up-regulation of the anti-apoptotic bcl-2 protein and down-regulation of the pro-apoptotic Bax protein in L02-Ad-AE cells analyzed by Western blot. (d) Increase of phosphorylated AKT and Bad in L02-Ad-AE cells, suggesting the activation of PI3K/AKT signaling and inhibition of apoptosis. (Li et al., 2011). 
A
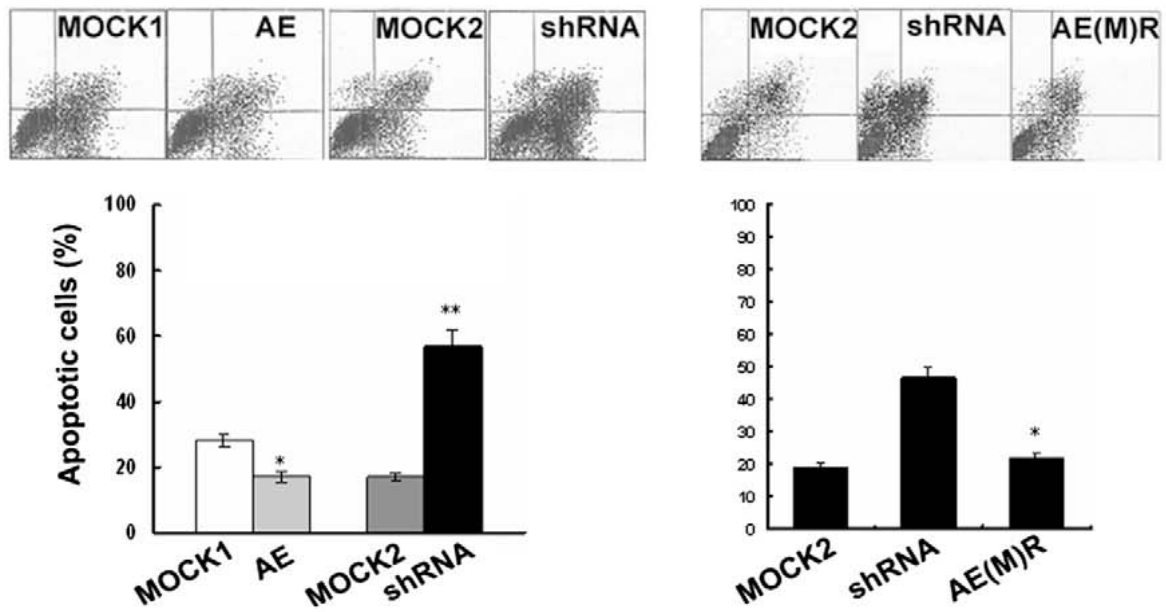

B

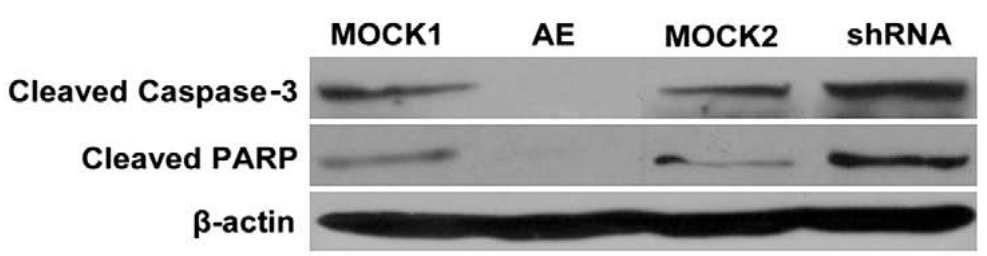

Fig. 13. Up-regulation and down-regulation of LAPTM4B-35 respectively inhibits and promotes HepG2 cells apoptosis induced by adriamycin. (a) Adriamycin $(15 \mu \mathrm{g} / \mathrm{ml})$ induced apoptosis analyzed by FACS in HepG2-AE, HepG2-shRNA, HepG2-AE (M)R cells and corresponding controls. $\mathrm{P}<0.05$ : HepG2-AE cells vs. HepG2-mock1, and HepG2-AE (M) R vs. HepG2-shRNA, P < 0.001: HepG2-shRNA vs. HepG2-mock2. (b) Analysis of cleaved caspase- 3 and PARP by Western blot, demonstrating activation and inhibition of the apoptotic pathway respectively by up-regulation and down-regulation of LAPTM4B-35 in HepG2 cells.

\subsection{Over-expression of LAPTM4B-35 enhances migration and invasion}

Cell migration and invasion are prerequisites for metastasis. Using LAPTM4B-35 overexpressing HCC cells, including HLE-AE, HepG2-AE and BEL7402-AE cells, it has been demonstrated that the cells over-expressing LAPTM4B-35 display enhanced capacity for migration and invasion (XR. Liu et al., 2009; H. Yang et al., 2010c). Conversely downregulation of endogenous LAPTM4B-35 by RNAi inhibits migration and invasion of HCC cells (Figure 14, H. Yang et al., 2010c). In addition, the matrix metal proteases (MMP2 and MMP9) and urine plasminogen activator (uPA) which are key proteases for cancer cell invasion, are up-regulated and/or activated by over-expression of LAPTM4B-35 (L. Zhou et al., 2010). 
A
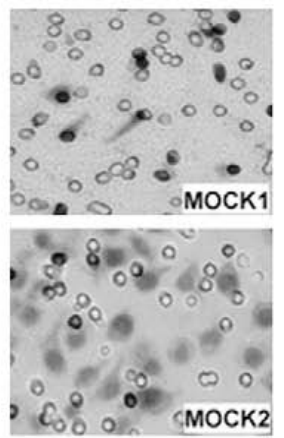

$\mathrm{B}$
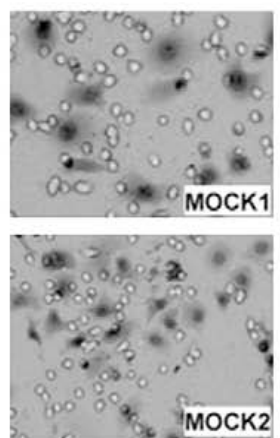
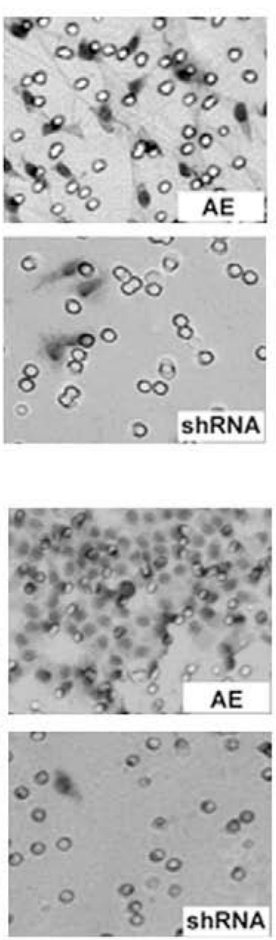
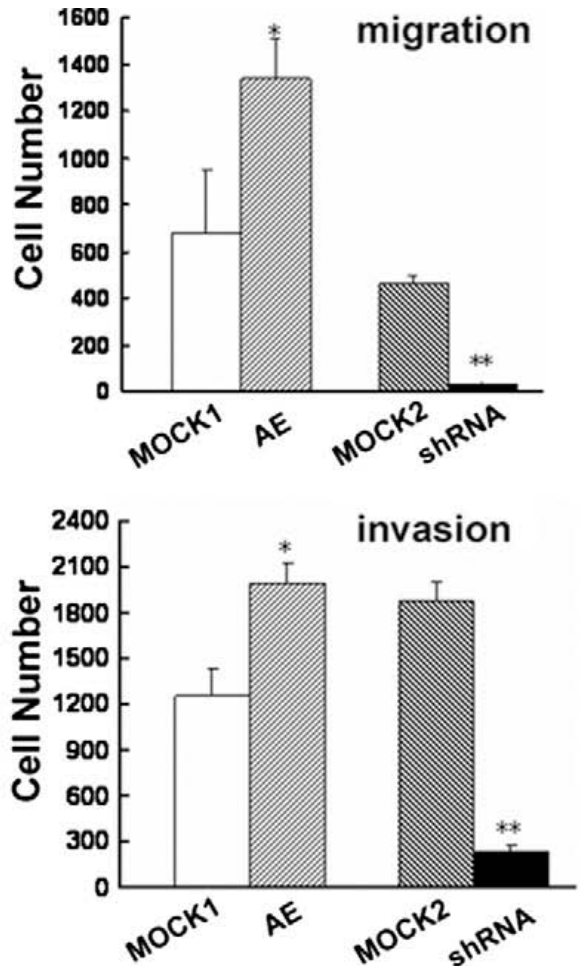

Fig. 14. Over-expression of LAPTM4B-35 promotes migration and invasion. The left panels: (a) Cell migration measured by Boyden chamber assay was promoted and inhibited, respectively, by over-expression (upper panel) and knockdown (lower panel) of LAPTM4B35 in HepG2 cells. (b) Cell invasion measured by Boyden chamber assay in the presence of Matrigel is promoted and inhibited, respectively, by over-expression (upper panel) and knockdown (lower panel) of LAPTM4B-35 in HepG2 cells. The right panels are the histograms showing number of migratory and invasive cells. ${ }^{*} \mathrm{P}<0.05$ : HepG2-AE vs. HepG2-mock1; ${ }^{* *} \mathrm{P}<0.001$ HepG2-shRNA vs. HepG2-mock2.

\subsection{Over-expression of LAPTM4B-35 motivates multi-drug resistance}

Multi-drug resistance is a significant obstacle in cancer chemotherapy. We have demonstrated ( $\mathrm{L} \mathrm{Li}$ et al., 2010) that drug efflux and resistance to multiple drugs including doxorubicin, paclitaxel and cisplatin, are enhanced in LAPTM4B-35 over-expressing L02-AE cells that were originally derived from normal liver (Figure 15a), whereas these phenomena are reversed in LAPTM4B-35 down-regulated HeLa-RNAi cells (Figure 15b). In addition, the drug retention in HeLa-RNAi cells was significantly more than in HeLa-Mock control cells (Figure 15c). These findings imply multi-drug resistance is promoted by over-expression of LAPTM4B-35 (L Li et al., 2010). At the same time, LAPTM4B associated multi-drug resistance has also been demonstrated in breast cancer (Hu, 2009; Y.Li., 2010) and ovarian cancer (Yin, 2011a) by other research groups. Y Li et al. (2010) demonstrated that amplification of LAPTM4B and YWHAZ, which was shown by fluorescence in situ 
A
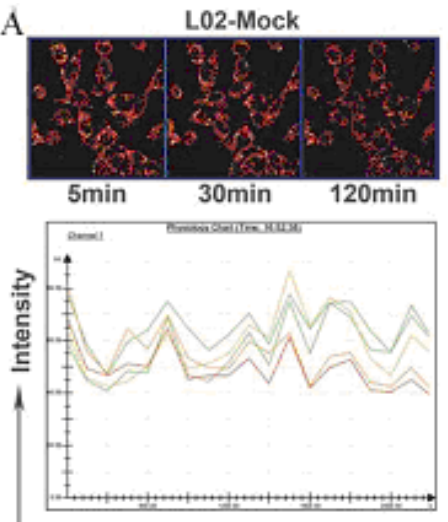

Times(s)

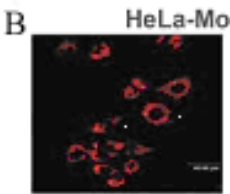

$0 \mathrm{~min}$

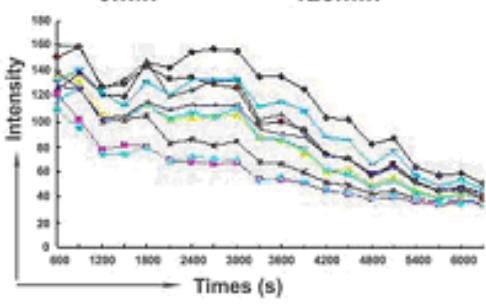

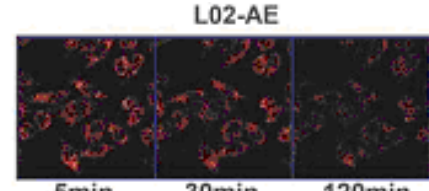

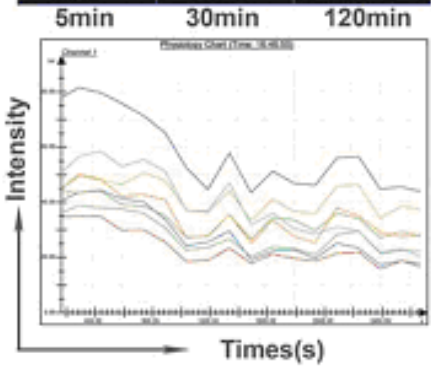

HeLa-RNAi

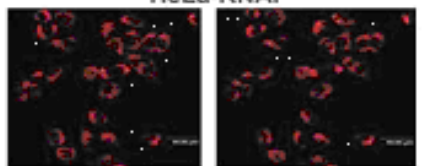

$0 \mathrm{~min}$

$120 \mathrm{~min}$

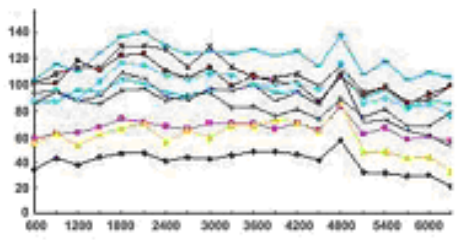

D
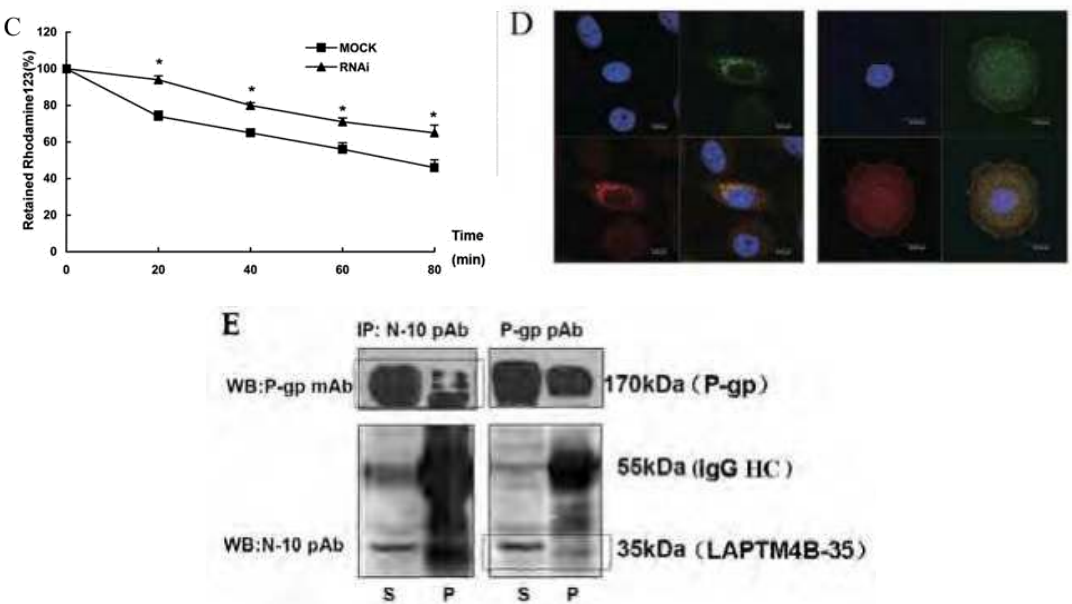

Fig. 15. LAPTM4B-35 motivates multi-drug efflux by interaction with P-gp. (a) Upper panel: The LAPTM4B-35 over-expressing L02-AE cells show faster efflux of Rhodamine-123 as 
compaired with L02-Mock control cells. (b) Upper panel: The LAPTM4B-35 knocking down HeLa-RNAi cells show slower drug efflux as compaired with HeLa-Mock cells. The drug efflux was measured by Laser Scanning Confocal Microscopy (LSCM). Lower Panels of (a) and (b): The fluorescence intensity in each cell was monitored and recorded separately as each curve. (c) The Rhodamine-123 retention in HeLa-RNAi cells is significantly greater than that of HeLa Mock control cells $\left({ }^{*} \mathrm{P}<0.05\right)$. The time course of Rhodamine-123 retention in LAPTM4B-35 knocking down HeLa-RNAi cells and HeLaMock cells was analyzed by a FACS. (d) Left panel: LAPTM4B-35 is co-localized with P-gp (MDR1) mainly at endomembrane organelles in HeLa cells (Bar, $8 \mathrm{~mm}$ ). Right panel: LAPTM4B-35 is co-localized with P-gp mainly on plasma membrane in PC-3 cells which are spreading onto fibronectin substrate (Bar, $20 \mathrm{~mm}$ ). Co-localization of LAPTM4B-35 and P-gp was analyzed by Triple-staining immunofluorescence and LSCM. Yellow color is the overlapping of green (P-gp) and red (LAPEM4B-35) signals. (e) Interaction of LAPTM4B-35 with P-gp analyzed by Co-immunoprecipitation (Co-IP). The antibodies used for immuno-precipitation were anti-LAPTM4B-35-N10pAb (left panel) and anti-P-gp $\mathrm{pAb}$ (right panel). The antibodies used for immuno-blotting (Western blot) were anti-P-gp $\mathrm{mAb}$ (upper panel) and anti-LAPTM4B-35-N10pAb (lower panel). Interaction of LAPTM4B-35 with P-gp is demonstrated by Co-IP analysis of both sets marked with a box with red line. (L Li et al., 2010)

hybridization (FISH), contributes to chemotherapy resistance of breast cancer. Hu et al (2009) reported activation of MTDH and LAPTM4B (which are localized at the same gene locus) determined by gain of $8 \mathrm{q} 22$ via comparative genomic hybridization (CGH) promotes chemoresistance and metastasis of breast cancer. These observations are consistent with our current results. All these studies indicate that LAPTM4B plays an important role in multidrug resistance. Our study on the mechanism indicates that there is a molecular interaction and co-localization between LAPTM4B-35 and P-gp (MDR1). P-gp is a classic transporter that results in multi-drug resistance by enhancing drug efflux from cancer cells. Interestingly, the co-localization presents at the plasma membrane when cancer cells are spreading onto extracellular matrix component fibronectin, but at intracellular membrane compartments (this may mainly involve endosomes and lysosomes based on the distribution pattern) when cancer cells are not spreading onto extracellular matrix component (Figure 15d). Since P-gp trafficking between lysosomes and plasma membrane plays a critical role in multi-drug resistance (Fu et al., 2004, 2007), our results suggest that LAPTM4B-35 may be a significant factor that is involved in the trafficking of LAPTM4B35 and P-gp between plasma membrane and intracellular compartments in giving rise to multi-drug resistance. The detailed mechanism for this combined trafficking remains to be further studied. Additionally, increasing numbers of experiments have recently shown that activation of the PI3K/AKT signaling pathway can regulate or enhance multi-drug resistance (Abdul-Ghani et al., 2006; Knuefermann et al., 2003; McCubrey et al., 2006; Tazzari et al., 2007). Our study indicates that PI3K/AKT signaling pathway is remarkably activated by over-expression of LAPTM4B-35 (see section 3.6). Accordingly, PI3K inhibitors can inhibit AKT phosphorylation/activation and increase the sensitivity of cancer cells over-expressing LAPTM4B-35 to doxorubicin, paclitaxel and cisplatin (L Li et al., 2010). Overall, our data provide new insight into the molecular mechanisms of multidrug resistance and open a novel avenue for overcoming multi-drug resistance in chemotherapy by targeting LAPTM4B-35. 


\subsection{Over-expression of LAPTM4B-35 activates a signaling network associated with carcinogenesis}

Over-expression of LAPTM4B-35 in liver and hepatocellular carcinoma cell lines enhances a wide range of malignant cellular and molecular phenotypes. The mechanism for the wide variety of roles played by over-expression of LAPTM4B-35 is therefore of great interest and has provoked scientific attention. We believe the most likely mechanism which may account for the findings and phenotypic changes described above is the involvement of LAPTM4B35 in a signaling network. Based on the striking similarity of the proline-rich motifs at the Nterminus of LAPTM4B-35 and at the PI3K p85 $\alpha$ regulatory subunit, we first explored the effect of LAPTM4b-35 over-expression in HCC cells on activation of the PI3K/AKT signaling pathway. We found that LAPTM4b-35 up-regulation constantly activates PI3K/AKT signaling which has been shown by increased phosphorylation of AKT and its down stream signaling molecules (Figure 16a) in HepG2, BEL7402 HCC cells and HeLa cells (L Li et al., 2010; Yang et al., 2010). Furthermore, interaction between the proline-rich motif of LAPTM4B-35 N-terminal tail and the PI3K p $85 \alpha$ regulatory subunit is demonstrated by site-specific mutations, GST-pull down and co-immunoprecipitation (Co-IP) (L Li et al., 2010; XR Liu et al., 2009; and unpublished data). It is believed that binding of the prolinerich motif of LAPTM4B-35 to the SH3 domain of PI3K p85 $\alpha$ regulatory subunit may release the inhibitory effect of $\mathrm{p} 85 \alpha$ regulatory subunit of PI3K on kinase activity of the p110 catalytic subunit of PI3K. We then found that the phosphorylation of GSK3 $\beta$ and FOXO4, both of which are downstream signaling molecules of phosphorylated/activated AKT, is promoted by over-expression and attenuated by knockdown of LAPTM4B-35 in HepG2-AE cells (Figure 16a). Since GSK3 $\beta$ (Glycogen synthase kinase 3 beta) is inactivated after phosphorylation by AKT, as a result the phosphorylation of c-Myc and cyclinD1, which are the GSK3 $\beta$ down stream effectors, is thus diminished and increased, respectively, in LAPTM4B-35 over-expressing and knocking down cancer cells (Figure16b). Sequentially, because diminished phophorylation of c-Myc and cyclinD1 can lead to decrease of degradation by proteosomes via ubiqutination (Diehl J et al., 1998), as a result, c-Myc and cyclinD1 become more stable and thus accumulate in LAPTM4B-35 over-expressing cancer cells (Figure 10a and 16c) playing roles in carcinogenesis. On the other hand, FOXO4 is a transcription factor of p27. It is known that phosphorylation of FOXO4 may result in sequestration in the cytoplasm with resultant loss of its function (Medema et al., 2000). This may be the reason that expression of p27 is dramatically diminished by overexpression of LAPTM4B-35, but restored by its knockdown via RNAi (Figure 10a). Based on the fact that p27 is a member of the cyclin-dependent kinase inhibitor (CKI) family, and that restored expression of p27 may inhibit cell proliferation and thus be a favorable prognostic indicator for patients with HCC (M.Fiorentino et al., 2000), it is possible that clinical therapy using LAPTM4B-35 specific RNA interference may improve the prognosis of HCC patients. In addition, the integrin/FAK, RTK/Ras/ERK and Wnt signaling pathways are also activated by LAPTM4B-35 over-expression (unpublished data). It has been demonstrated via GST-pull down, Co-IP and site-specific mutations that LAPTM4B-35 can interact with several signal molecules in cytoplasm and plasma membrane, such as PI3K p85 $\alpha, \mathrm{FAK}$, integrins ( $\alpha 5$ and $\alpha 6$ ) and RTKs (for example IGF1R), and so on. Taken together, it is evident that LAPTM4B35 over-expression activates a signaling network, consisting of at least four signaling pathways which are closely associated with hepatocarcinogenesis, metastasis and multidrug resistance (Whittaker, 2010). It is proposed that LAPTM4B-35 may act as an organizer 
or platform of signal molecules which functions at signal network upstream. Further investigation of this point will greatly improve our understanding on hepatocarcinogenesis, metastasis and recurrence of hepatocellular carcinoma, and will thus provide novel strategies for targeted chemotherapy of hepatocellular carcinoma.

Overall our studies demonstrate there is a relationship of LAPTM4B-35 over-expression with up-regulation of proliferation-promoting proteins and down-regulation of proliferation-inhibiting proteins in hepatocellular carcinoma cells. This relationship is mediated by a signaling network.

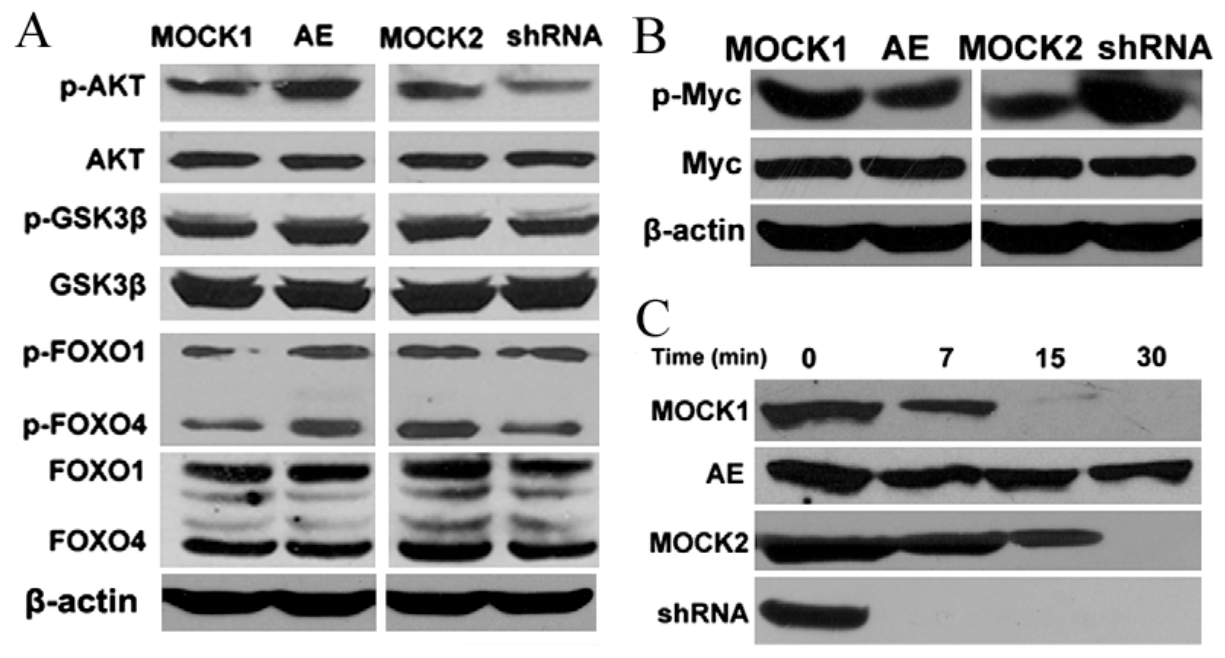

Fig. 16. LAPTM4B-35 over-expression activates a signaling network. (a) Over-expression and knockdown of LAPTM4B-35, respectively, activates and inhibits PI3K/AKT signaling pathway shown by Western blot. AKT activation is generally evaluated by its phosphorylations at S 308 and S473 resulting from PI3K activation through its phosphorylating product PIP3 and two additional kinases. GSK3 $\beta$ and FOXO4 are both down stream effectors of AKT and thus phosphorylated by this activated kinase. (b) Phosphorulated c-Myc (p-Myc) is diminished and increased, respectively, by overexpression and knockdown in HepG2 cells. (c) Degradation of c-Myc is respectively diminished by over-expression of LAPTM4B-35 and enhanced by knowdown of LAPTM4B35. This experiment was performed via cycloheximide pulse-chase assay, in which stably transfected HepG2 cells were treated with $50 \mu \mathrm{g} / \mathrm{ml}$ of cycloheximide to inhibit biosynthesis of proteins and harvested at 0, 7, 15 and 30 min., then Western blot with anti-Myc Ab was performed to evaluate degradation of c-Myc. The results indicates that up-regulation of LAPTM4B-35 enhances stability of c-Myc, and vice versa.

\subsection{LAPTM4B-24 up-regulation induces apoptosis and autophagocytosis, and abolishes carcinogenicity of hepatocellular carcinoma cells}

LAPTM4B-24 is an isoform encoded by the LAPTM4B gene. It is a truncated form of LAPTM4B-35 by lacking a 91 amino acid sequence at the N-terminus (Shao et al., 2003). We have demonstrated that up-regulation of LAPTM4B-24 via transfection with plasmids 
pcDNA-BE or infection with replication-deficient adenovirus Ad-BE induces apoptosis and autophagocytosis of HCC cells, as well as associated cellular and molecular alterations. At the same time LAPTM4B-24 up-regulated HCC cells lose its carcinogenicity (unpublished data). These studies indicate that LAPTM4B-24 plays an antagonistic role in hepatocarcinogenesis.

In summary, the LAPTM4B-35 plays pivotal roles in keeping cell survival, proliferation, migration and invasion, and so on; whereas LAPTM4B-24 plays critical roles in regulating programmed cell death, including apoptosis and autophagocytosis. The expressive equilibrium of LAPTM4B-35 and LAPTM4B-24 maintains physiological homeostasis of cells. Destroy of this equilibrium would lead to diseases. Up-regulation of LAPTM4B-35 leads to oncogenesis, while up-regulation of LAPTM4B-24 may plays a role in cancer regression.

\section{LAPTM4B gene and the encoded LAPTM4B-35 protein are predicted to be novel markers for diagnosis of hepatocellular carcinoma}

\subsection{LAPTM4B-35 is predicted to be a novel marker for diagnosis, pathological grading and progression-monitoring of hepatocellular carcinoma}

We have demonstrated via Western blot and immuno-histochemistry that the expression levels of LAPTM4B-35 in HCC tissues show a significant positive correlation with pathological grade (Figure 17a, and Table 1 and 3), intrahepatic and extrahepatic metastasis (figure 17b), TNM stage (Table 1 and 3) and recurrence of HCC (Table 3), and show a negative correlation with overall and disease-free postoperative survival (Figure 17c). Upon multivariate analysis, elevated expression of LAPTM4B-35 was found to be an independent

\begin{tabular}{|c|c|c|c|c|}
\hline \multirow[t]{2}{*}{ Variables } & \multirow[t]{2}{*}{ Patiens } & \multicolumn{2}{|c|}{ LAPTM4B-35 expression } & \multirow[t]{2}{*}{$P^{\prime \prime}$} \\
\hline & & Low & High & \\
\hline All cases & 71 & & & \\
\hline \multicolumn{5}{|l|}{ Tumor size } \\
\hline$\delta \mathrm{cm}$ & 38 & 10 & 28 & 0.710 \\
\hline$\geq 5 \mathrm{~cm}$ & 33 & 10 & 23 & \\
\hline \multicolumn{5}{|c|}{ Portal vein invasion } \\
\hline No & 52 & 20 & 32 & 0.001 \\
\hline Yes & 19 & 0 & 19 & \\
\hline \multicolumn{5}{|c|}{ Serum AFP level } \\
\hline$<25 \mathrm{ng} / \mathrm{ml}$ & 35 & 13 & 22 & 0.097 \\
\hline$\geq 25 \mathrm{ng} / \mathrm{ml}$ & 36 & 7 & 29 & \\
\hline \multicolumn{5}{|l|}{ TNM stage } \\
\hline I-II & 25 & 14 & 11 & $<0.001$ \\
\hline III-IV & 46 & 6 & 40 & \\
\hline \multicolumn{5}{|l|}{ Recurrence } \\
\hline No & 17 & 13 & 4 & $<0.001$ \\
\hline Yes & 54 & 7 & 47 & \\
\hline
\end{tabular}

Table 3. Expressive level of LAPTM4B-35 in HCC tissues shown by IHC shows significant positive correlation to portal vein invasion, TNM staging and recurrence. (H.Yang et al., 2010b). 

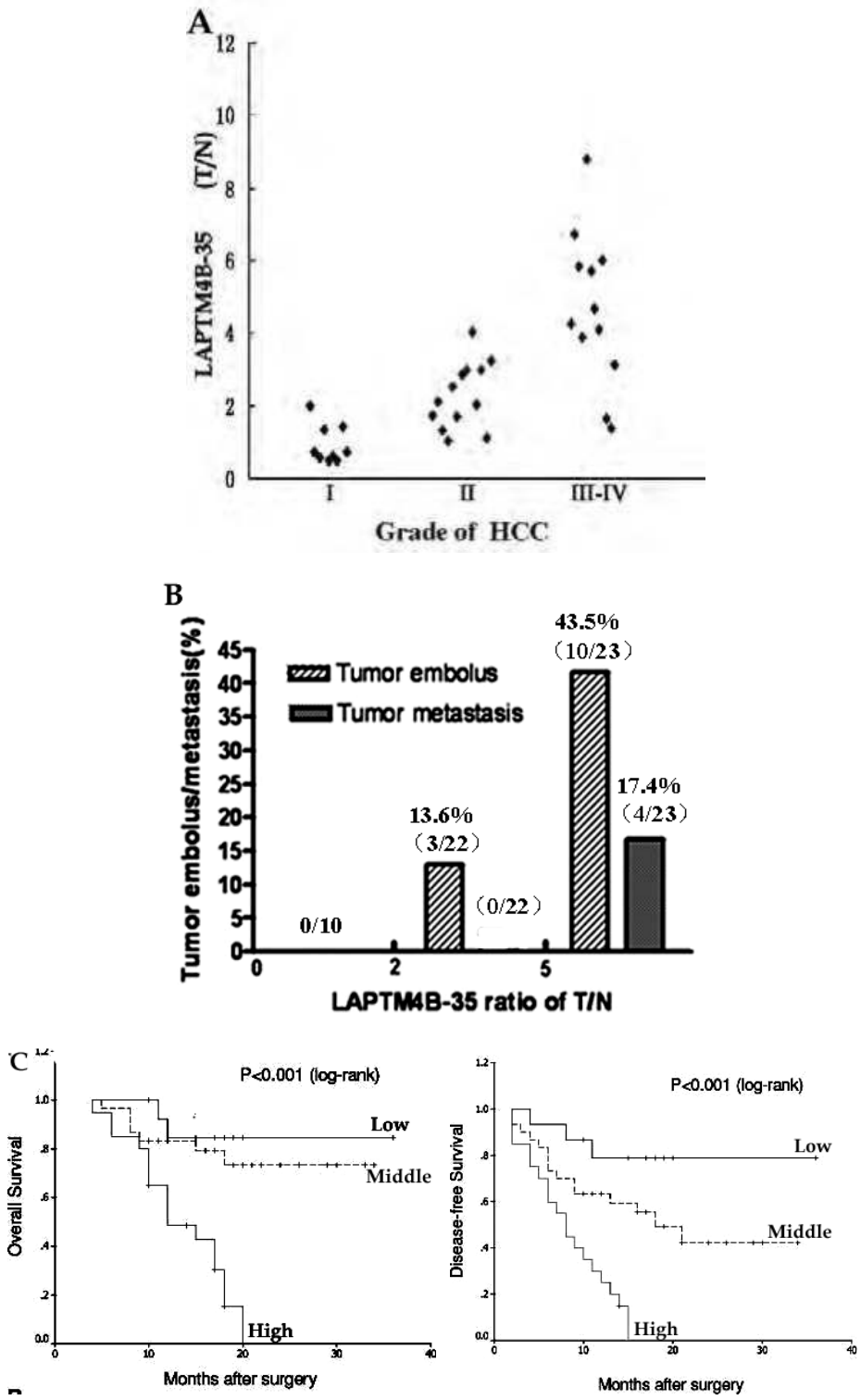

Fig. 17. Correlation of LAPTM4B-35 expression levels in HCC tissues with pathological grading, metastasis and postoperative survival time. (a) Level of LAPTM4B-35 protein in HCCs shows positive correlation with pathological grade. (b) LAPTM4B-35 expressions in HCCs from 65 patients were divided into three groups: "Low," "Mediate," and "High." Level of LAPTM4B-35 in HCC shows significant positive correlation with intrahepatic and extrahepatic metastasis. ${ }^{*} \mathrm{P}<0.05$. (c) Levels of LAPTM4B-35 in HCCs show significantly negative correlation with overall (left) and disease-free (right) postoperative survival of cancer patients. (H.Yang et al., 2010a, 2010b). 
prognostic factor for hepatocellular carcinoma (Yang et al., 2010a, 2010b). In our preliminary study we found that about $40 \%$ of paired non-cancerous liver tissues in the same HCC patients express elevated LAPTM4B mRNA (JJ. Liu et al., 2000). We believe that the noncancerous liver tissue of the same one patient with HCC is representing the precancer status to variant extents. We therefore suggest that LAPTM4B-35 may be an important and novel biomarker for early diagnosis, mornitoring of progression, prediction of metastasis and recurrence of HCC, as well as for evaluating prognosis of HCC patients. In addition, we have demonstrated that LAPTM4B-35 protein can be released into the blood in the form of exosomes. The levels of LAPTM4B-35 in serum of HCC patients are significantly higher than those from normal individuals (unpublished data).

Moreover, a number of other cancers also show the similar association of LAPTM4B-35 with clinical and pathological criteria. Cancers which have been evaluated include gallbladder cancer (L. Zhou et al., 2007), cholangiocarcinoma (L. Zhou et al., 2008), ovarian cancer (Y Yang, 2008; Yin et al., 2011a, 2011b), cervical cancer (Meng et al., 2010), and endometrial cancer (Meng et al., 2010). It is likely that LAPTM4B-35 may also find use as a biomarker for other solid cancers.

\subsection{LAPTM4B mRNA expressed in circulating tumor cells can be used for predicting metastasis}

It is well known that circulating cancer cells have more highly metastatic potential. We found that the highly metastatic cancer cell lines derived from one cancer mass express higher levels of LAPTM4N-35 than syngenic cancer cell lines of low metastatic potential (XR. Liu et al., 2003). We have determined that the LAPTM4B mRNA expressed by a few circulating HCC cells can be measured by nested RT-PCR and real time RT-PCR. Therefore, testing LAPTM4B mRNA in circulating HCC cells with real time RT- PCR shows promise for application of prediction and evaluation of metastasis occurring at an early stage.

\section{LAPTM4B gene and the encoded LAPTM4B-35 protein as a novel therapeutic targets}

\subsection{Adjusting the expression equilibrium between LAPTM4B-35 and LAPTM4B-24 can be a novel strategy for biotherapy of hepatocellular carcinoma}

As described in section 3, the expression equilibrium of LAPTM4B-35 and LAPTM4B-24 plays a pivotal role in maintaining the physiological status of cells. LAPTM4B-35 promotes resistance to apoptosis, deregulation of proliferation, enhancement of migration and invasion, and multi-drug resistance. On the other hand, up-regulation of LAPTM4B-24 increases sensitivity to induction of apoptosis and autophagocytosis. As we have demonstrated in our laboratory knockdown of the high expression of the endogenous cancer-promoting protein LAPTM4B-35 via RNAi, or elevating the relatively low expression of the cancer-inhibiting protein LAPTM4B-24 by transgenic therapy can significantly inhibit cancer growth and metastasis and thus may be applicable in cancer biotherapy. Therefore, we suggest knockdown of LAPTM4B-35 expression via RNAi or microRNA, and/or upregulation of LAPTM4B-24 expression may provide effective strategies for treatment of HCC and possibly other cancers. 


\subsection{LAPTM4B-35 is a novel therapeutic target for neoadjuvant chemotherapy}

Neoadjuvant chemotherapy generally targets one or more molecules in signaling pathways. As discussed in section 3, over-expression of LAPTM4B-35 promotes hepatocarcinogenesis, faster growth of human HCC xenografts and metastasis in mice, and leads to anti-apoptosis, deregulation of proliferation, and enhancement of migration and invasion and multi-drug resistance. In addition, over-expression of LAPTM4B-35 leads to accumulation of a number of cell cycle promoting proteins and survival proteins, and results in down-regulation of a number of cell cycle inhibiting proteins and proapoptotic proteins by activating a signaling network including at least 4 different signaling pathways. Based on our studies in vitro and in vivo, it is clear that LAPTM4B-35 has significant potential as an important novel target for cancer treatment. Recently, we have screened out a few small molecules from various libraries containing nearly 2000 chemically synthetic compounds. These small molecules are able to kill a variety of cancer cells that over-express the LAPTM4B gene and LAPTM4B-35 protein in vitro, but do not harm the normal fetal liver cells which do not over-express this gene. One of these small molecules has been demonstrated to inhibit growth and metastasis of human HCC xenografts in nude mice, and results in cellular and molecular alterations opposite to those that result from over-expression of LAPTM4B-35 in HCC cells. Some of these active small molecules have been demonstrated to be inhibitors of tyrosine kinase (unpublished data).

In summary, this gene and the proteins it encodes harbor great potential for application in cancer biotherapy and chemotherapy.

\section{Conclusion and prospective}

LAPTM4B gene and its encoding LAPTM4B-35 protein are over-expressed in $87 \%$ of HCC and some solid cancers with varying frequencies. The level of LAPTM4B-35 expression in HCC tissues shows significant positive correlation with pathological grade, intrahepatic and extrahepatic metastasis, and recurrence, and negative correlation with overall and diseasefree postoperative survival of cancer patients, and is thus an independent prognostic factor for HCC. Over-expression of LAPTM4B-35 promotes malignant transformation of cell lines from normal tissues, including human liver tissue. This result implies that over-expression of the LAPTM4B gene and LAPTM4B-35 protein may play pivotal roles in hepatocarcinogenesis and progresion. Up-regulated LAPTM4B-35 promotes faster growth and metastasis of human HCC xenografts in nude mice, and results in resistance to apoptosis, deregulation of proliferation, and enhancement of migration and invasion, as well as multi-drug resistance. In addition, overexpression of LAPTM4B-35 leads to accumulation of a number of oncoproteins encoded by oncogenes and down-regulation of a number of tumor suppressing proteins. In contrast, knockdown of endogenous LAPTM4B35 via RNA interfering leads to significant inhibition of growth and metastasis of human HCC xenografts in nude mice, and reversion of the cellular and molecular malignant phenotypes. The extensive effects caused by LAPTM4B-35 over-expression result from its function in activation of a signaling network, including at least 4 signaling pathways that are commonly known to be associated with hepatocarcinogenesis (K. Breuhahn, 2010; Takigawa, 2008; XH , 2011). Taken together, our studies suggests that LAPTM4B-35 is a key molecule which functions upstream of a cancer associated signaling network and plays pivotal roles in hepatocarcinogenesis, progression, metastasis, multi-drug resistance and 
recurrence. Conversely, up-regulation of LAPTM4B-24, an truncated isoform that lacks a 91 amino acid sequence at the N-terminus of LAPTM4B-35, promotes apoptosis and autophagocytosis, and therefore plays an antagonistic role in hepatocarcinogenesis. Overall, these data provide new insight into genes and proteins that are potentially important in the pathogenesis of liver carcinoma. This harbors great potential for future application as novel biomarkers for cancer diagnostics, pathological grading, progression monitoring and prognosis, and as novel molecular targets providing new strategies for biotherapy and chemotherapy of HCC.

Up-regulation of expression of the LAPTM4B gene and LAPTM4B-35 protein occur in a broad range of human solid cancers and are often associated with poor prognosis, indicating there is a common key role for this oncogentic gene and protein in cancer development.

Although LAPTM4B gene and the encoded LAPTM4B protein have been the subject of more than a decade of study, there are still important issues which remain to be resolved. These include (1) the underlying mechanism(s) responsible for up-regulation of the LAPTM4B gene and LAPTM4B-35 protein in neoplastic transformation; (2) the possibility LAPTM4B may be associated with cancer stem cells. Our previous studies indicate that LAPTM4B-35 over-expressing cancer cells possess characteristics typical of cancer stem cells, including apoptosis-resistance, unlimited renewal capacity, metastatic potential and multi-drug resistance. In addition, a study by Lee et al., (2010) suggests that LAPTM4B together with Gp49a, Sox4, and CD34 genes may be "stemness-related" genes in a primitive hematopoietic progenitor cell line EML, and this study demonstrates that these genes are preferentially expressed in hematopoietic stem cells and down-regulated in mature hematopoietic cells. Study of whether LAPTM4B-35 protein is a marker of cancer stem cells is therefore warranted; (3) as it has been shown in our study that LAPTM4B protein localizes at late endosomes, lysosomes, mitochondria, and the plasma membrane (unpublished data), it may be supposed that trafficking of LAPTM4B protein in cells would be very important for execution of its functions, thus it is worthwhile studying when and how LAPTM4B-35 and LAPTM4B-24 trafficking functions in normal cells and in cancer cells; (4) the detailed molecular mechanism for enhancing function of multi-drug resistance of $p$-gp (MDR1) by LAPTM4B-35 over-expression; (5) the underlying mechanism for metastasis promoted by LAPTM4B-35 over-expression; (6) the detailed molecular mechanism for signaling network activation by LAPTM4B-35 over-expression in HCC; (7) the sensitivity and specificity of LAPTM4B mRNA and LAPTM4B-35 protein as a marker for HCC diagnosis; (8) the underlying molecular mechanism for which targeted chemotherapy of small compounds depends on LAPTM4B-35 over-expression.

\section{Acknowledgment}

I sincerely appreciate all the members of my laboratory who have worked on and are currently working on LAPTM4B for their valuable contributions, and I appreciate the work which has been done by other groups on this topic. These studies of LAPTM4B have been supported by grants from 211 and 985 Foundation of Peking University, National High-tech R\&D Program (863 Program) (2006AA02A305), 248 major R\&D program of Beijing (H020220020310), NNSFC (90408018) and the Creative Drugs Foundation from Beijing Fangzheng Group. 
I would also like to sincerely thank Professor Da Long Ma and his Colleges who have given me great encourage and help, and Dr. Michael A. McNutt who has provided great help in use of the English language.

\section{References}

Abdul-Ghani R., Serra V., Gyo“ rffy B., Ju“ rchott K., Solf A., Dietel M.et al. (2006). The PI3K inhibitor LY294002 blocks drug export from resistant colon carcinoma cells overexpressing MRP1. Oncogene, Vol.25, pp.1743-1752.

Adra CN., Zhu S., Ko JL., Guillemot JC., Cuervo AM., Kobayashi H., Horiuchi T., Lelias JM., Rowley JD, Lim B. (1996) LAPTM5: a novel lysosomal-associated multispanning membrane protein preferentially expressed in hematopoietic cells. Genomics. 35(2):328-37.

Breuhahn K. \& Schirmacher P. (2010). Signaling networks in human hepatocarcinogenesis-novel aspects and therapeutic options. Prog Mol Biol Transl Sci. Vol.97, pp.251-77.

Buendia MA. (2002) Genetic alterations in hepatoblastoma and hepatocellular carcinoma: common and distinctive aspects. Med Pediatr Oncol. Vol.39, No.5, pp.530-5.

Longerich T., Mueller MM., Breuhahn K., Schirmacher P., Benner A. \& Heiss C. (2011). Oncogenetic tree modeling of human hepatocarcinogenesis. Int J Cancer. Mar 11. doi: 10.1002/ijc.26063. [Epub ahead of print]

Cheng XJ., Xu W., Zhang QY. \& Zhou RL. (2008). Relationship between LAPTM4B gene polymorphism and susceptibility of colorectal and esophageal cancers. Annals of Oncology, Vol.19, No.3, pp.527-32

Deng LJ., Zhang QY., Liu B. \& Zhou RL. (2005). Relationship between LAPTM4B gene polymorphism and susceptibility of lung cancer, J Peking University (health Sciences), Vol.37, No.3, pp.302-305

Diehl JA., Cheng M., Roussel MF. \& Sherr CJ. (1998). Glycogen synthase kinase-3beta regulates cyclin D1 proteolysis and subcellular localization. Genes Dev, Vol.12, pp.499-3511.

Fiorentino M., Altimari A., D'Errico A., Cukor B., Barozzi C., Loda M. \& Grigioni WF. (2000). Acquired expression of p27 is a favorable prognostic indicator in patients with hepatocellular carcinoma. Clin. Cancer Res. Vol.6, No.10, pp.3966-3972.

Fu D., Bebawy M., Kable EP. and Roufogalis BD. (2004a). Dynamic and intracellular trafficking of P-glycoprotein-EGFP fusion protein: Implications in multidrug resistance in cancer. Int J Cancer, Vol.109, pp.174-181

He J., Shao GZ \& Zhou RL. (2003), Effects of the novel gene, LAPTM4B, high expression in hepatocellular carcinoma on cell proliferation and tumorigenesis of NIH3T3 cells, J Peking University (health Sciences), Vol.35, No.4, (December 2002), pp.348-352, ISSN1671-167X

Hogue DL., Ellison MJ., Young JD. \& Cass CE. (1996). Identification of a novel membrane transporter associated with intracellular membranes by phenotypic complementation in the yeast Saccharomyces cerevisiae. J Biol Chem., Vol.271, No.16, pp.9801-8

Hogue DL., Kerby L. \& Ling V.A. (1999). Mammalian lysosomal membrane protein confers multidrug resistance upon expression in Saccharomyces cerevisiae. J Biol Chem, Vol.274, No.18, pp.12877-82 
Hu, GH., Chong, RA., Yang, QF., Wei, Y., Blanco, MA., Li, F., , Reiss, M., Au, JL.-S., Haffty, BG. \& Kang, YB. (2009). MTDH Activation by 8q22 Genomic Gain Promotes Chemoresistance and Metastasis of Poor-Prognosis Breast Cancer. Cancer Cell, Vol.15, pp.9-20.

Huang J., Sheng HH., Shen T., Hu YJ., Xiao HS., Zhang Q., Zhang QH. \& Han ZG. (2006). Correlation between genomic DNA copy number alterations and transcriptional expression in hepatitis B virus-associated hepatocellular carcinoma. FEBS Lett. Vol.580, No.15, pp.571-81.

Kasper G., Vogel A., Klaman I, et al. (2005). The human LAPTM4b transcript is upregulated in various types of solid tumours and seems to play a dual functional role during tumour progression. Cancer Letters, Vol.224, pp.93-103

Knuefermann C., Lu Y., Liu B., Jin W., Liang K., Wu L. et al. (2003). HER2/PI-3K/Akt activation leads to a multidrug resistance in human breast adenocarcinoma cells. Oncogene 22: 3205-3212.

Lee H M., Zhang H., Schulz V., Tuck DP., \& Forget BG. 2010, Downstream targets of HOXB4 in a cell line model of primitive hematopoietic progenitor cells. Blood, Vol.116, No.5, pp.720-730

Li, L., Wei, XH., Pan, YP., Shan, Y., Li, HC., Yang, H., Pang, Y., Xiong, FX., Shao, GZ. \& Zhou RL. (2010). LAPTM4B : A novel cancer-associated gene motivates multi-drug resistance through efflux and activating PI3K/Akt signaling. Oncogene, Vol.29, No.43, pp.5785-95.

Li Y., Zou L., Li Q., Haibe-Kains B., Tian1 R., Li,Y., Desmedt C., Sotiriou C., Szallasi Z., Iglehart JD., Richardson AL. \& Wang ZC. (2010). Amplification of LAPTM4B and YWHAZ contributes to chemotherapy resistance and recurrence of breast cancer. Nature Medicine; Vol.16, pp. 214-8.

Liu J., Zhang J., Zhang N., Rui J., Jin C. \& Zhou R. (2000), Identification of new hepatocellular carcinoma genes by fluorescent differential display. J Peking University (health Sciences), Vol.32, No.5, (October 2000), pp. 411-14, ISSN 1000-1530.

Liu XR., Zhou RL. , Zhang QY.., Zhang Y., Shao GZ., Jin YY., Zhang S., Lin M., Rui JA. \& Ye DX. (2003). Identification and characterization of LAPTM4B encoded by a human hepatocellular carcinoma-associated novel gene, J Peking University (health Sciences), Vol.35, No.4, (May 2003), pp.340-347, ISSN1671-167X

Liu XR., Zhou RL., Zhang QY., Zhang Y., Jin YY., Lin M, Rui JA. \& Ye DX. (2004). Structure analysis and expressions of a novel tetratransmembrane protein, LAPTM4B, associated with hepatocellular carcinoma. World J Gestroenterology, Vol.10, No.11, pp.1555-1559.

Liu XR., Xiong FX., Wei XH., Yang H. \& Zhou RL. (2009). LAPTM4B-35, a Novel Tetratransmembrane Protein and its PPRP Motif Play Critical Roles in Proliferation and Metastatic Potential of HCC Cells, Cancer Sci, Vol.100, No.12, pp.2335-2340.

Liu Y., Zhang QY., Qian N. \& Zhou RL. (2007). Relationship between LAPTM4B gene polymorphism and susceptibility of gastric cancer, Annals of Oncology, Vol.18, No.2, pp.311-316

Longerich T., Mueller MM., Breuhahn K., Schirmacher P., Benner A., Heiss C., Marchio A., Meddeb M., Pineau P., Danglot G., Tiollais P., Bernheim A. \& Dejean A. (1997). Recurrent chromosomal abnormalities in hepatocellular carcinoma detected by 
comparative genomic hybridization. Genes Chromosomes Cancer. Vol.18, No.1, pp.5965.

Martin E. H. (2005). Tetraspanin functions and associated microdomains, Nature Reviews Molecular Cell Biology, Vol.6, pp. 801-811

McCubrey JA., Steelman LS., Abrams SL., Lee JT., Chang F., Bertrand FE., Navolanic PM., Terrian DM., Franklin RA., D'Assoro AB., Salisbury JL., Mazzarino MC., Stivala F. \& Libra M. (2006). Roles of the RAF/MEK/ERK and PI3K/PTEN/AKT pathways in malignant transformation and drug resistance. Adv Enzyme Regul. Vol.46, pp.24979.

Medema RH., Kops GJ., Bos JL., Burgering BM. (2000). AFX-like Forkhead transcription factors mediate cell-cycle regulation by Ras and PKB through p27kip1. Nature, Vol.404, No.6779, pp.782-787.

Meng FL., Luo C., Hu YL., Yin MZ., Lin M., Lou G. \& Zhou RL. (2010a). Over-expression of LAPTM4B-35 in Cervical Carcinoma. Int J Gynecol Pathol, Vol.29, No.6, pp.587-93.

Meng FL., Yin MZ., Hong-tao Song, T Hua Yang, t Ge Lou, * \& Zhou RL. (2010b). LAPTM4B35 Over-expression Is an Independent Prognostic Marker in Endometrial Carcinoma. Int J Gynecol Cancer, Vol.20, No.5, pp.745-50.

Meng F., Song H., Luo C., Yin M., Xu Y., Liu H., Zhou R., \& Lou G. (2011). Correlation of LAPTM4B polymorphisms with cervical carcinoma. Cancer, Vol.117, No.12, pp.2652-8.

Ouchida R., Kurosaki T. \& Wang JY. (2010). A role for lysosomal-associated protein transmembrane 5 in the negative regulation of surface B cell receptor levels and B cell activation. J Immunol. Vol.185, No.1, pp.294-301.

Peng C., Zhou RL., Shao GZ., Rui JA., Wang SB., Lin M., Zhang S \& Gao ZF. (2005). The expressions of LAPTM4B-35, a novel tetratransmembrane protein, in cancers and the correlation with the differentiation status of HCC. World J Gestroenterology, Vol.11, No.18, pp.2704-2708. ISSN 1007-9324

Sears R., Nuckolls F., Haura E., Taya Y., Tamai K. \& Nevins J R. (2000). Multiple Rasdependent phosphorylation pathways regulate Myc protein stability. Genes Dev;Vol.14, pp.2501-2514.

Seimiya M., O-Wang J., Bahar R., Kawamura K., Wang Y., Saisho H. \& Tagawa M. (2003). Stage-specific expression of Clast6/E3/LAPTM5 during B cell differentiation: elevated expression in human B lymphomas. Int J Oncol. Vol..22, No.2, pp.301-4.

Shao GZ., Zhou RL., Zhang QY., Zhang Ye., Liu JJ.,. Rui JA., Wei XH. \& Ye DX. (2003). Molecular cloning and characterization of LAPTM4B, a novel gene upregulated in hepatocellular carcinoma. Oncogene, Vol.22, pp.5060-5069

Takigawa Y. \& Brown AM. (2008). Wnt signaling in liver cancer. Curr Drug Targets. Vol.9, No.11, pp.1013-24.

Tazzari PL., Cappellini A., Ricci F., Evangelisti C., Papa V., Grafone T.et al. (2007). MRP1 \& $\mathrm{PI} 3 \mathrm{~K} / \mathrm{Akt}$ multidrug resistance-associated protein 1 expression is under the control of the phosphoinositide 3 kinase/Akt signal transduction network in human acute myelogenous leukemia blasts. Leukemia, Vol.21, pp.427-438.

Vergarajauregui S., Martina JA. \& Rosa Puertollano. (2011). LAPTMs regulate lysosomal function and interact with mucolipin 1: new clues for understanding mucolipidosis type IV. Journal of Cell Science, Vol.124, pp.459-468 
Wang XH., Meng XW., Sun X., Liu BR., Han MZ., DU YJ., Song YY. \& Xu W. (2011). Wnt/ $\beta$ catenin signaling regulates MAPK and Akt1 expression and growth of hepatocellular carcinoma cells. Neoplasma. Vol.58, No.3, pp.239-44.

Whittaker S., Marais R. \& Zhu AX., 2010, The role of signaling pathways in the development and treatment of hepatocellular carcinoma. Oncogene, Vol.29, pp.4989-5005

Yang, H., Xiong, FX., Lin, M., Qi, RZ., Liu, ZW., Rui, JA., Su, J. \& Zhou, RL. (2010a). LAPTM4B-35 Is a Novel Diagnostic Marker and a Prognostic Factor of Hepatocellular Carcinoma. J Surgical Oncology, Vol.101, pp.363-369.

Yang H, Xiong FX, Lin M, Yang Y, Nie X and Zhou RL. (2010b) LAPTM4B-35 overexpression is a risk factor for tumor recurrence and poor prognosis in hepatocellular carcinoma, J Cancer Res Clin Oncol., Vol.136, pp.275-281.

Yang H., Xiong F., Wei X., Yang Y., McNutt MA. \& Zhou RL. (2010c). Over-expression of LAPTM4B-35 promotes growth and metastasis of hepatocellular carcinoma in vitro and in vivo. Cancer Lett, Vol.294, pp.236-244.

Yang Y., Yang H. , Mcnutt MA., Xiong FX., Nie X., Li L. \& Zhou RL. (2008). LAPTM4B over-expression is an independent prognostic marker in ovarian carcinoma, Oncology Reports, Vol.20, No.5, pp.1077-1083.

Yin M., Li C., Li X., Lou G., Miao B., Liu X., Meng F., Zhang H., Chen X., Sun M., Ling Q. \& Zhou RL. (2011a). Over-expression of LAPTM4B is associated with poor prognosis and chemotherapy resistance in stages III and IV epithelial ovarian cancer. J Surg Oncol. Vol.104, No.1, pp.29-36.

Yin M., Xu Y., Lou G., Hou Y., Meng F., Zhang H., Li C. \& Zhou RL. (2011b). LAPTM4B over-expression is a novel predictor of epithelial ovarian carcinoma metastasis. Int J Cance, Vol.129, No.3, pp.629-35

Zhou L., He XD., Chen J., Cui QC., Qu Q., Rui JA. \& Zhao YP. (2007) Overexpression of LAPTM4B-35 closely correlated with clinicopathological features and postresectional survival of gallbladder carcinoma. Eur J Cancer. Vol.43, No.4, pp.809-15.

Zhou L., He XD., Cui QC., Zhou WX., Qu Q., Zhou RL., Rui JA. \& Yu JC. (2008), Expression of LAPTM4B-35: A novel marker of progression, invasiveness and poor prognosis of extrahepatic cholangiocarcinoma. Cancer Lett. Vol.264, No.2, pp.209-217.

Zhou L., He XD., Yu JC., Zhou RL., Yang H., Qu Q. \&, Rui JA. (2010a). Over-expression of LAPTM4B promotes growth of gallbladder carcinoma cells in vitro. Am J Surg, Vol.199, No.4, pp.515-21.

Zhou L., He XD., Yu JC., Zhou RL., Xiong FX., Qu Q., Rui JA. (2010b). Expression of LAPTM4B in gallbladder carcinoma cells: the role in invasive potential. Hepatogastroenterology, Vol. 57, No.98, pp.207-11.

Zhou L., He XD., Yu JC., Zhou RL., Shan Y. \& Rui JA. (2011). Overexpression of LAPTM4B35 attenuates epirubucin-induced apoptosis of gallbladder carcinoma GBC-SD cells. Surgery, Vol. 150, No.1, pp.25-31 


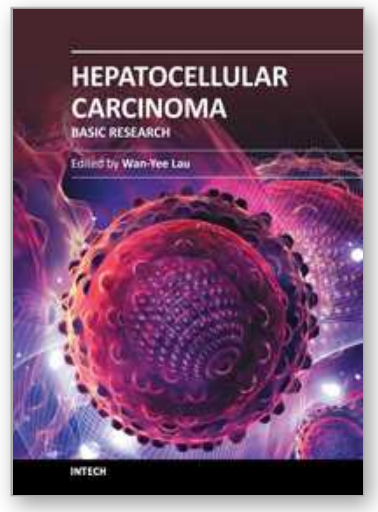

\author{
Hepatocellular Carcinoma - Basic Research \\ Edited by Dr. Joseph W.Y. Lau
}

ISBN 978-953-51-0023-2

Hard cover, 402 pages

Publisher InTech

Published online 10, February, 2012

Published in print edition February, 2012

Hepatocellular Carcinoma represents a leading cause of cancer death and a major health problem in developing countries where hepatitis $B$ infection is prevalent. It has also become increasingly important with the increase in hepatitis $C$ infection in developed countries. Knowledge of hepatocellular carcinoma has progressed rapidly. This book is a compendium of papers written by experts to present the most up-to-date knowledge on hepatocellular carcinoma. This book deals mainly with the basic research aspect of hepatocellular carcinoma. The book is divided into three sections: (I) Biomarkers / Therapeutic Target; (II) Carcinogenesis / Invasion / Metastasis; and (III) Detection / Prevention / Prevalence. There are 18 chapters in this book. This book is an important contribution to the basic research of hepatocellular carcinoma. The intended readers of this book are scientists and clinicians who are interested in research on hepatocellular carcinoma. Epidemiologists, pathologists, hospital administrators and drug manufacturers will also find this book useful.

\title{
How to reference
}

In order to correctly reference this scholarly work, feel free to copy and paste the following:

Rou Li Zhou (2012). LAPTM4B: A Novel Diagnostic Biomarker and Therapeutic Target for Hepatocellular Carcinoma, Hepatocellular Carcinoma - Basic Research, Dr. Joseph W.Y. Lau (Ed.), ISBN: 978-953-51-00232, InTech, Available from: http://www.intechopen.com/books/hepatocellular-carcinoma-basic-research/1laptm4b-a-novel-gene-associated-with-hepatocellular-carcinoma

\section{INTECH}

open science | open minds

\section{InTech Europe}

University Campus STeP Ri

Slavka Krautzeka 83/A

51000 Rijeka, Croatia

Phone: +385 (51) 770447

Fax: +385 (51) 686166

www.intechopen.com

\section{InTech China}

Unit 405, Office Block, Hotel Equatorial Shanghai

No.65, Yan An Road (West), Shanghai, 200040, China 中国上海市延安西路65号上海国际贵都大饭店办公楼405单元 Phone: +86-21-62489820

Fax: $+86-21-62489821$ 
(C) 2012 The Author(s). Licensee IntechOpen. This is an open access article distributed under the terms of the Creative Commons Attribution 3.0 License, which permits unrestricted use, distribution, and reproduction in any medium, provided the original work is properly cited. 